\title{
On the emergence of large clusters of acoustic power sources at the onset of thermoacoustic instability in a turbulent combustor
}

\author{
Abin Krishnan $^{1,} \dagger$, R. I. Sujith ${ }^{1}$, Norbert Marwan ${ }^{2}$ and Jürgen Kurths ${ }^{2,3}$ \\ ${ }^{1}$ Indian Institute of Technology Madras, Chennai 600036, India \\ ${ }^{2}$ Potsdam Institute for Climate Impact Research, 14412 Potsdam, Germany \\ ${ }^{3}$ Department of Physics, Humboldt University, 10115 Berlin, Germany
}

(Received 25 August 2018; revised 21 May 2019; accepted 23 May 2019; first published online 9 July 2019)

In turbulent combustors, the transition from stable combustion (i.e. combustion noise) to thermoacoustic instability occurs via intermittency. During stable combustion, the acoustic power production happens in a spatially incoherent manner. In contrast, during thermoacoustic instability, the acoustic power production happens in a spatially coherent manner. In the present study, we investigate the spatiotemporal dynamics of acoustic power sources during the intermittency route to thermoacoustic instability using complex network theory. To that end, we perform simultaneous acoustic pressure measurement, high-speed chemiluminescence imaging and particle image velocimetry in a backward-facing step combustor with a bluff body stabilized flame at different equivalence ratios. We examine the spatiotemporal dynamics of acoustic power sources by constructing time-varying spatial networks during the different dynamical states of combustor operation. We show that as the turbulent combustor transits from combustion noise to thermoacoustic instability via intermittency, small fragments of acoustic power sources, observed during combustion noise, nucleate, coalesce and grow in size to form large clusters at the onset of thermoacoustic instability. This nucleation, coalescence and growth of small clusters of acoustic power sources occurs during the growth of pressure oscillations during intermittency. In contrast, during the decay of pressure oscillations during intermittency, these large clusters of acoustic power sources disintegrate into small ones. We use network measures such as the link density, the number of components and the size of the largest component to quantify the spatiotemporal dynamics of acoustic power sources as the turbulent combustor transits from combustion noise to thermoacoustic instability via intermittency.

Key words: turbulent reacting flows, vortex dynamics

\section{Introduction}

In most practical applications, such as in the power generation and aerospace industries, combustion happens in a confined space. Unsteady combustion tends to

$†$ Email address for correspondence: abin.roja@gmail.com 
drive the acoustic modes of the confinement (Hegde, Reuter \& Zinn 1988). As a consequence, combustors are susceptible to the phenomenon of thermoacoustic instability, which occurs when a positive feedback is established between the acoustic field and the heat release rate oscillations. Thermoacoustic instability is characterized by large-amplitude periodic pressure oscillations, and leads to the failure of system components due to increased mechanical and thermal stresses (McManus, Poinsot \& Candel 1993; Candel 2002; Juniper \& Sujith 2018). Lord Rayleigh (1878) explained that acoustic energy is added to the system if the heat release rate occurs in phase with the pressure oscillations. When the acoustic driving overcomes the damping present in the system, the combustor transits to thermoacoustic instability (Putnam 1971).

In order to understand the transition from stable combustion to unstable combustion, i.e. thermoacoustic instability, researchers have used tools from dynamical systems theory. Early studies in this direction considered the onset of thermoacoustic instability as a loss of stability of a fixed point via Hopf bifurcation (Lieuwen 2002; Ananthkrishnan, Deo \& Culick 2005). This description works very well for laminar systems. However, for turbulent combustors, the description is incomplete.

Thermoacoustic oscillations in turbulent combustors have also been studied from a stochastic viewpoint in time domain analysis. In this approach, the background turbulent fluctuations in the combustors are modelled as a noise term. Lieuwen \& Banaszuk (2005) examined the effect of modelling the background turbulent fluctuations both as additive and parametric excitation sources on the combustor's stability boundaries. They observed that additive and parametric excitation sources had a quantitative and qualitative impact, respectively, on the combustor dynamics. Clavin, Kim \& Williams (1994) modelled the effect of turbulence on heat release rate as parametric noise that appeared in the wave equation as a multiplicative noise term. The Fokker-Planck equation for the evolution of the transition probability density function for the amplitude of pressure oscillation predicted erratic bursts, as observed in liquid rocket motors. Recently, Noiray \& Schuermans (2013a) modelled combustion noise as additive white noise and analysed supercritical bifurcations in the corresponding stochastic differential equations. Using the same approach, Noiray \& Schuermans (2013b) explained the switching between the standing and spinning azimuthal thermoacoustic modes in annular combustors.

Nair et al. (2013) remarked that the low-amplitude aperiodic pressure oscillation characterizing stable combustion (also known as combustion noise in the community) in a turbulent combustor is not exactly 'noise' in the usual sense. Through a series of tests of determinism (the Kaplan-Glass test and the 0-1 test for chaos), the authors suggested that combustion noise is deterministic and displays a chaotic behaviour. Later, Tony et al. (2015), through a more detailed surrogate analysis, confirmed that combustion noise is indeed deterministic chaos. These studies show that the low-amplitude aperiodic pressure oscillations generated in a turbulent reactive flow field characterizing stable combustion do not correspond to a stable fixed point.

In a turbulent combustor, the transition from combustion noise to thermoacoustic instability occurs via a dynamical state known as intermittency (Nair, Thampi \& Sujith 2014). Combustion noise is characterized by low-amplitude aperiodic pressure fluctuations. In contrast, thermoacoustic instability is characterized by high-amplitude periodic pressure oscillations. Intermittency, the in-between state, has the signature of both combustion noise and thermoacoustic instability. It is characterized by bursts of high-amplitude periodic oscillations embedded amidst epochs of low-amplitude aperiodic oscillations, in an apparently random manner. Following this discovery 
by Nair et al. (2014), many other studies also observed intermittency en route to thermoacoustic instability in turbulent combustors. A review of this can be found in Pawar \& Sujith (2018). Intermittency is also observed en route to lean blowout in turbulent combustors (Gotoda et al. 2011, 2012, 2014; Unni \& Sujith 2015).

These studies concentrated only on the temporal dynamics during the transition to thermoacoustic instability. In the past, there have been studies that examined the spatiotemporal dynamics during the transition to thermoacoustic instability. Most of these studies, however, focused on the spatiotemporal dynamics only during combustion noise and thermoacoustic instability. These studies elucidated the emergence of large-scale coherent structures at the onset of thermoacoustic instability and the modification of the flame dynamics by these flow structures (Rogers \& Marble 1956; Smith \& Zukoski 1985; Poinsot et al. 1987; Schadow \& Gutmark 1992; Coats 1996; Shanbhogue 2008; Schwing, Sattelmayer \& Noiray 2011; Emerson et al. 2012).

Recently, there have been a few studies that have focused on the spatiotemporal dynamics during the transition to an impending thermoacoustic instability. Unni \& Sujith (2017) investigated the flame dynamics during the intermittency route to thermoacoustic instability in a turbulent combustor with a bluff body stabilized flame. During combustion noise, the flame is stabilized along both the outer and the inner shear layer by the recirculation zone downstream of the dump plane and the stagnation point flow behind the bluff body, respectively. During the periodic epochs of intermittency, the flame, stabilized along both the outer and inner shear layers, oscillates in a periodic fashion. A roll-up of the flame along the outer shear layer occurs due to the periodic shedding of ring vortices from the dump plane. During the aperiodic epochs of intermittency, the flame tip oscillates in an aperiodic manner. On the contrary, during thermoacoustic instability, a stronger roll-up of the outer shear layer occurs due to the emergence of periodic large-scale coherent structures downstream of the dump plane, imparting a strong periodic behaviour to the flame front.

Apart from the flow and the flame dynamics, some studies explored the coupling between the pressure and the heat release rate oscillations during the intermittency route to thermoacoustic instability. Pawar et al. (2017) used synchronization theory and described the transition to thermoacoustic instability as mutual synchronization of two non-identical oscillators, namely the acoustic field of the combustor and the heat release rate oscillations. During intermittency, the authors observed intermittent phase synchronization, wherein these two oscillators are synchronized during the periodic epochs and desynchronized during the aperiodic epochs of their oscillations.

Mondal, Unni \& Sujith (2017), by calculating the instantaneous phase between the pressure and the heat release rate oscillations, examined the spatial distribution of acoustic power sources in the flow field during the three different dynamical states of combustor operation, namely combustion noise, intermittency and thermoacoustic instability. During combustion noise, the authors observed that the acoustic power production occurs in a spatially incoherent manner. In contrast, during thermoacoustic instability, the acoustic power production occurs in a spatially coherent manner over large regions. However, during intermittency, both incoherent and coherent patches of acoustic power sources coexist simultaneously, resembling a chimera-like state. Kheirkhah et al. (2017), following a similar procedure, observed that the phase difference between the pressure and the heat release rate oscillations changes during the growth and decay of pressure oscillations during intermittency.

However, to date, studies have not focused on describing how large regions of spatially coherent acoustic power sources emerge at the onset of thermoacoustic 
instability from a state of spatially incoherent acoustic production observed during combustion noise. The present study tries to find the answer to the above question by examining the spatiotemporal dynamics of acoustic power sources during the intermittency route to thermoacoustic instability in a turbulent combustor.

A turbulent flow induces a large number of degrees of freedom in the thermoacoustic system. The thermoacoustic system in a turbulent combustor involves the interplay of a number of processes such as molecular mixing, turbulent transport, chemical kinetics and acoustic waves operating over a range of length and time scales. The complex interaction of these processes over such a large number of degrees of freedom gives rise to a rich spatiotemporal dynamics, with multifractal chaotic behaviour on the one hand (during combustion noise) and ordered periodic behaviour on the other (during thermoacoustic instability). This prompts us to consider the thermoacoustic system in a turbulent combustor as a complex system.

The complex systems approach is a new approach in science to studying how the interaction between its constituent parts gives rise to the collective behaviour of a system (Bar-Yam 1997). This approach is providing a new perspective on the understanding of the physical, biological, ecological and social universe. One of the efficient tools for studying such complex systems is complex networks (Barabási 2011). In complex network theory, the components of a complex system are considered as nodes and the interactions between the nodes are represented as links. The discovery of small world networks (Watts \& Strogatz 1998) and scale-free networks (Barabási \& Albert 1999) paved the way for the birth of complex network theory.

Based on ideas from graph theory and statistical physics, complex network theory allows a detailed and quantitative investigation of the interplay between network topology and dynamics of the interacting elements (Albert \& Barabási 2002). Researchers are now trying to understand how an enormous network of interacting dynamical systems behaves collectively, given the systems' individual dynamics and coupling architecture. Complex network theory has found applications in different frontiers of science and engineering such as brain research, epidemics, climatology, sociology, economics, physiology, computer science and transport engineering (Strogatz 2001; Barabasi 2003; Boccaletti et al. 2006; Donges et al. 2009; Newman 2010; Barthélemy 2011).

Recently, complex network theory was introduced for the analysis of thermoacoustic systems. Murugesan \& Sujith (2015), using the visibility algorithm (Lacasa et al. 2008) to construct networks from the time trace of acoustic pressure, brought to light the scale-free nature of combustion noise. They observed that as a turbulent combustor transits from combustion noise to thermoacoustic instability, the topology of the network changes from scale-free to regular, reflecting the emergence of order from disorder. Okuno, Small \& Gotoda (2015) used cycle networks and phase-space networks to show the high dimensional nature of thermoacoustic instability. Godavarthi et al. (2017) used recurrence networks to study the topology of the phase space corresponding to the different dynamical states of combustor operation. Gotoda et al. (2017) showed the presence of a small-world nature in the recurrence network constructed from the combustion state close to lean blowout.

Most real-world complex systems have nodes and edges embedded in space. Transportation networks, internet, social and contact networks, power grids and neural networks are all examples where space is relevant, and just studying the network topology without taking into consideration the spatial information of the nodes gives incomplete and sometimes misleading results. Characterizing and understanding the 
structure and dynamics of spatial networks is thus vital in obtaining deeper insights in the study of complex systems (Barthélemy 2011).

Spatial network analysis has also been used in the study of fluid mechanical systems. In climatology, researchers explored the statistical interdependence between the time series of an observable, such as precipitation, at two different locations (spatially embedded nodes) to construct spatial networks (Tsonis \& Roebber 2004; Malik et al. 2012; Molkenthin et al. 2014). Recently, the area of turbulence research has also witnessed the application of spatial network analysis. Taira, Nair \& Brunton (2016) characterized a two-dimensional decaying isotropic turbulent flow field, obtained from direct numerical simulation, based on the vortical interaction between the fluid elements at different grid points using the Biot-Savart law. Scarsoglio, Iacobello \& Ridolfi (2016) identified coherent structures in the flow field using a correlation network based on the time series of the kinetic energy of a three-dimensional forced isotropic turbulent flow field obtained from direct numerical simulation.

Singh et al. (2017) extended the application of spatial network analysis to turbulent reactive flows. The authors developed a visibility algorithm for spatially embedded nodes for characterizing turbulent flames. The authors observed that the hubs of the network coincided with the folds in the flame front, which was attributed to the interaction of the flame front with turbulent eddies. Also, the flame front network was resilient against a random removal of nodes but susceptible to a targeted removal of hubs, thus opening up the possibility of control of turbulent flames using network theory. Recently, Unni et al. (2018) performed spatial network analysis, in a turbulent combustor with a bluff body stabilized flame, based on the correlation between the time series of local velocity obtained from particle image velocimetry (PIV). The authors identified the critical regions in the flow field using network centrality measures, during combustion noise, intermittency and thermoacoustic instability.

In the spatial network analysis discussed so far, the temporal evolution of the system under study was not taken into account. However, real-world complex systems inherently evolve over time. Time-varying network analysis accounts for this dynamical nature of connectivity (Tang et al. 2010; Holme \& Saramäki 2012; Nicosia et al. 2012). Time-varying spatial network analysis is used extensively in the area of brain research to analyse the dynamical functional connectivity of brain networks (Valencia et al. 2008; Dimitriadis et al. 2010; Doron, Bassett \& Gazzaniga 2012; Cabral, Kringelbach \& Deco 2017).

In the present paper, we perform time-varying spatial network analysis to examine the spatiotemporal dynamics of acoustic power sources during the different dynamical states of combustor operation. In time-varying network studies, the time series of an observable is divided into different windows or segments and a spatial network is constructed in each of these segments. In the current study, however, we construct a spatial network at each instant of time. To that end, we perform simultaneous acoustic pressure measurement, high-speed chemiluminescence imaging and PIV in a backward-facing step combustor with a bluff body stabilized flame at different equivalence ratios. For each of these different equivalence ratios, spatial networks are constructed at each time instant by connecting only the locations of acoustic power production. In this way, we examine the dynamics of acoustic power sources, both temporally and spatially, at different dynamical states as the turbulent combustor transits from combustion noise to thermoacoustic instability via intermittency.

The rest of the paper is organized as follows. The details of the experimental setup and the diagnostic techniques used are provided in $\S 2$. In $\S 3$, we describe the methodology of network construction. In $\S 4$, we discuss the results. Finally, in $\S 5$, the major findings are summarized. 


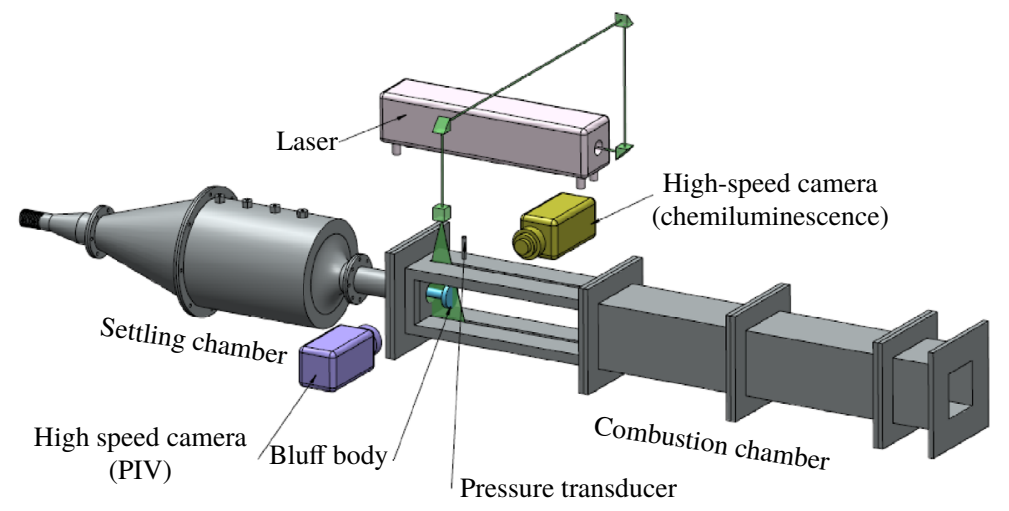

FIgURE 1. (Colour online) The schematic of the experimental set-up used in the current study. The design of the combustor was adapted from Komarek \& Polifke (2010).

\section{Experimental set-up}

We perform experiments in a backward-facing step combustor with a bluff body as the flame-holding device at high Reynolds number. The experimental set-up, as shown in figure 1, mainly consists of three parts - a settling chamber, a burner and a rectangular combustion chamber. More details regarding the experimental set-up can be found in Nair et al. (2013).

The air used for combustion enters the test section through the settling chamber. A blowdown mechanism is used to supply air from high-pressure tanks. A moisture separator removes the water content present in the compressed air before it enters the settling chamber. The settling chamber removes the fluctuations in the inlet air and hence decouples its effect on the spatiotemporal dynamics in the combustion chamber. Liquefied petroleum gas (LPG, $60 \%$ butane and $40 \%$ propane) is used as the fuel in the experiments. The fuel is injected into the burner, at $120 \mathrm{~mm}$ upstream of the bluff body, through four radial holes of $1.7 \mathrm{~mm}$ diameter using a central shaft. A circular disk of $2 \mathrm{~mm}$ thickness and $40 \mathrm{~mm}$ diameter with 300 holes of $1.7 \mathrm{~mm}$ diameter is located $30 \mathrm{~mm}$ downstream of the fuel injection location to act as a flashback arrestor. The combustion chamber consists of a sudden expansion from the circular burner of $40 \mathrm{~mm}$ diameter to a square cross-section of $90 \mathrm{~mm} \times 90 \mathrm{~mm}$ and a length of $1100 \mathrm{~mm}$. The partially premixed fuel-air mixture is spark-ignited in the recirculation zone of the dump plane using an $11 \mathrm{kV}$ ignition transformer. A circular disk (bluff body) of $40 \mathrm{~mm}$ diameter and $10 \mathrm{~mm}$ thickness is used for flame stabilization. The bluff body is attached at the end of the central shaft and is located at a distance of $45 \mathrm{~mm}$ downstream of the backward-facing step.

The control parameter in the experiment is the equivalence ratio $\left(\phi=\left(\dot{m}_{f u e l}\right)\right.$ $\left.\left.\dot{m}_{\text {air }}\right)_{\text {actual }} /\left(\dot{m}_{\text {fuel }} / \dot{m}_{\text {air }}\right)_{\text {stoichiometry }}\right)$, which is varied by keeping the fuel flow rate constant at 30 SLPM and increasing the air flow rate. The air and fuel flow rates are measured using mass flow controllers (Alicat Scientific, MCR Series, 100 SLPM model for fuel flow, 2000 SLPM for air flow; uncertainty is $\pm(0.8 \%$ of reading $+0.2 \%$ of full scale)). Throughout the experiments, the flow is turbulent ( $R e$ ranges from $1.9 \times 10^{4}$ to $3 \times 10^{4}$, with an uncertainty ranging from \pm 314 to \pm 400$)$. The unsteady pressure is measured using a piezoelectric transducer (PCB 103B02, with a sensitivity of $223.4 \mathrm{mV} \mathrm{kPa}^{-1}$ and an uncertainty of $\pm 0.15 \mathrm{~Pa}$ ), located at a distance of $40 \mathrm{~mm}$ downstream of the dump plane at a sampling frequency of $10 \mathrm{kHz}$ for $3 \mathrm{~s}$. A pair 
of quartz windows $(400 \mathrm{~mm} \times 90 \mathrm{~mm} \times 10 \mathrm{~mm})$ is provided in the side walls of the combustion chamber to facilitate optical diagnostics. A high-speed CMOS camera (Phantom-v 12.1) is used to capture the $\mathrm{OH}^{*}$ chemiluminescence from the flame, with a narrow band filter centred at $308 \mathrm{~nm}(12 \mathrm{~nm}$ FWHM). The flame images are recorded at a sampling frequency of $2 \mathrm{kHz}$, with an exposure time of $500 \mu \mathrm{s}$ and a resolution of 800 pixels $\times 600$ pixels. The camera is outfitted with a $50 \mathrm{~mm}$ ZEISS camera lens at $f / 2$ aperture. A total of 5034 images is acquired for each equivalence ratio that covers the transition of the turbulent combustor from combustion noise to thermoacoustic instability via intermittency.

We seed the flow with $\mathrm{TiO}_{2}$ particles (Kronos, product 1071) of diameter $1 \mu \mathrm{m}$ to obtain the Mie scattering images for the high-speed PIV. A single-cavity double-pulsed $\mathrm{Nd}$ :YLF laser (Photonics) with a wavelength of $527 \mathrm{~nm}$ and a pulse duration of $10 \mathrm{~ns}$ is used to illuminate the particle seeded flow. The repetition rate of each of the two pulses is $2 \mathrm{kHz}$. A second high-speed CMOS camera (Photron FASTCAM SA4), synchronized with the laser, outfitted with a ZEISS camera lens of $100 \mathrm{~mm}$ focal length with an aperture at $f / 5.6$, is used to acquire the Mie scattering images at a frame rate of $1000 \mathrm{~Hz}$. The measurement domain with a resolution of 640 pixels $\times 480$ pixels, located at the centre span of the bluff body, has a physical dimension of length $57 \mathrm{~mm}$ and width $33 \mathrm{~mm}$. A bandpass optical filter centred at $527 \mathrm{~nm}$ is mounted in front of the camera to filter the flame and other background luminosity. A detailed description of the analysis of PIV with the post-processing algorithms that are used to detect spurious vectors can be found in Unni et al. (2018). Simultaneous acoustic pressure measurement, $\mathrm{OH}^{*}$ chemiluminescence imaging and PIV were performed for different equivalence ratios during the intermittency route to thermoacoustic instability.

\section{Network construction}

In order to study the spatiotemporal evolution of acoustic power sources, we perform simultaneous high-speed chemiluminescence $\left(\mathrm{OH}^{*}\right)$ imaging of the combustion zone, near the dump plane of the combustor, along with the acoustic pressure measurement at different equivalence ratios during the transition from combustion noise to thermoacoustic instability via intermittency. The $\mathrm{OH}^{*}$ chemiluminescence serves as a marker for the heat release rate from the flame as the $\mathrm{OH}^{*}$ intensity depends upon the chemical reaction rate (Hurle et al. 1968; Nori \& Seitzman 2008). Even though the flow field is three-dimensional inside the combustor, we analyse it in a two-dimensional plane focused on the central plane of the combustor, with a depth of focus of $9 \mathrm{~mm}$. The raw images of resolution 800 pixels $\times 600$ pixels are cropped to a physical dimension of $57 \mathrm{~mm} \times 33 \mathrm{~mm}$, located at a distance of $28 \mathrm{~mm}$ downstream of the dump plane. We chose this region for the spatiotemporal analysis as it depicts interesting flow and flame dynamics during the different regimes of combustor operation (Unni \& Sujith 2017).

We measure the unsteady pressure $p^{\prime}(t)$ at a point near the dump plane. Nair et al. (2014) reported that the pressure variation across the combustion zone, in the present experimental set-up, is very small. Hence, we assume the pressure to be constant in the measurement domain at a given instant of time. In order to characterize the spatiotemporal evolution of acoustic power sources during a dynamical state of combustor operation, we multiply the unsteady heat release rate field $\dot{q}^{\prime}(x, y, t)$, obtained from the $\mathrm{OH}^{*}$ chemiluminescence images, with the unsteady pressure at the corresponding time instant. In this manner, we obtain a series of $5026 p^{\prime} \dot{q}^{\prime}$ images for 
a particular state of combustor operation. This is repeated for different equivalence ratios as the combustor transits from combustion noise to thermoacoustic instability via intermittency.

The product of $p^{\prime}$ and $\dot{q}^{\prime}$ represents the local acoustic power (Putnam 1971; Poinsot $\&$ Veynante 2005). Each pixel of the $p^{\prime} \dot{q}^{\prime}$ image represents a local acoustic power source if the value of $p^{\prime} \dot{q}^{\prime}$ is positive or a local acoustic power sink if it is negative or zero. For the time-varying spatial network analysis, we consider each pixel of the $p^{\prime} \dot{q}^{\prime}$ image to be a node. Two nodes are connected if and only if the values of $p^{\prime} \dot{q}^{\prime}$ at these two nodes are positive. As we connect only those nodes where $p^{\prime} \dot{q}^{\prime}$ are positive, we track the spatiotemporal evolution of only the acoustic power sources. Since the turbulent combustor is a fluid mechanical system where energy transfer takes place locally amongst the fluid elements mainly due to convection, we consider only the nearest neighbours $n_{i}$ of a node (the distance between the nodes should not exceed $\sqrt{ } 2$ ) when establishing a link. This implies that a node can have a maximum of only eight links. The average value of $p^{\prime} \dot{q}^{\prime}$ at the two connecting nodes (i.e. where $p^{\prime} \dot{q}^{\prime}$ is positive and the distance between the nodes is less than or equal to $\sqrt{ } 2$ ) is considered as the weight of the link between them. At a given value of the control parameter, we obtain high-speed chemiluminescence images for a duration of $2.513 \mathrm{~s}$ (with a sampling frequency of 2000 frames per second). Hence, at a given value of the control parameter, we have 5026 high-speed flame images and therefore 5026 time-varying weighted spatial acoustic power networks. In this manner, we construct 5026 timevarying weighted spatial networks for each equivalence ratio.

In order to characterize the spatiotemporal evolution of acoustic power sources, we use one of the basic weighted network measures - the node strength (Boccaletti et al. 2006). The node strength $(s)$ of a given node $i$ is defined as the sum of the weights of the links of a node. It is given by

$$
s_{i}=\sum_{j \in n_{i}} w_{i j},
$$

where $i$ and $j$ represent nodes of the network, $w$ the weight of the link connecting $i$ and $j$ and $n_{i}$ the neighbourhood of the node $i$. A node having higher node strength implies that the links of the given node carry higher weights. Since, in the present study, the weight of the link refers to the average value of $p^{\prime} \dot{q}^{\prime}$ at the two connecting nodes, regions of high node strength represent regions of high local acoustic power production.

Unni \& Sujith (2017) observed that vortices play a significant role in determining the flame dynamics during combustion noise, intermittency and thermoacoustic instability, as mentioned in $\S 1$. Hence, it is imperative that we analyse the vorticity field along with the heat release rate field to understand better the spatiotemporal evolution of the local acoustic power sources. Thus, we construct time-varying weighted spatial networks from the vorticity field, obtained from the analysis of PIV, along with the time-varying local acoustic power networks. The region of interest (ROI) considered for the analysis of PIV is the same as the one used for high-speed chemiluminescence imaging. In the analysis of PIV, the ROI in the flow field is divided into a rectangular grid. Each of the cells of this rectangular grid is known as an interrogation window (Raffel, Willert \& Kompenhans 2007). From the analysis of PIV, we obtain a velocity vector in each of these interrogation windows at every time instant. We consider these interrogation windows as the nodes of the vorticity network. Two nodes are connected if and only if the vorticity values $(\omega)$ at these nodes are either both positive or both negative. This implies that two nodes $i$ and $j$ 

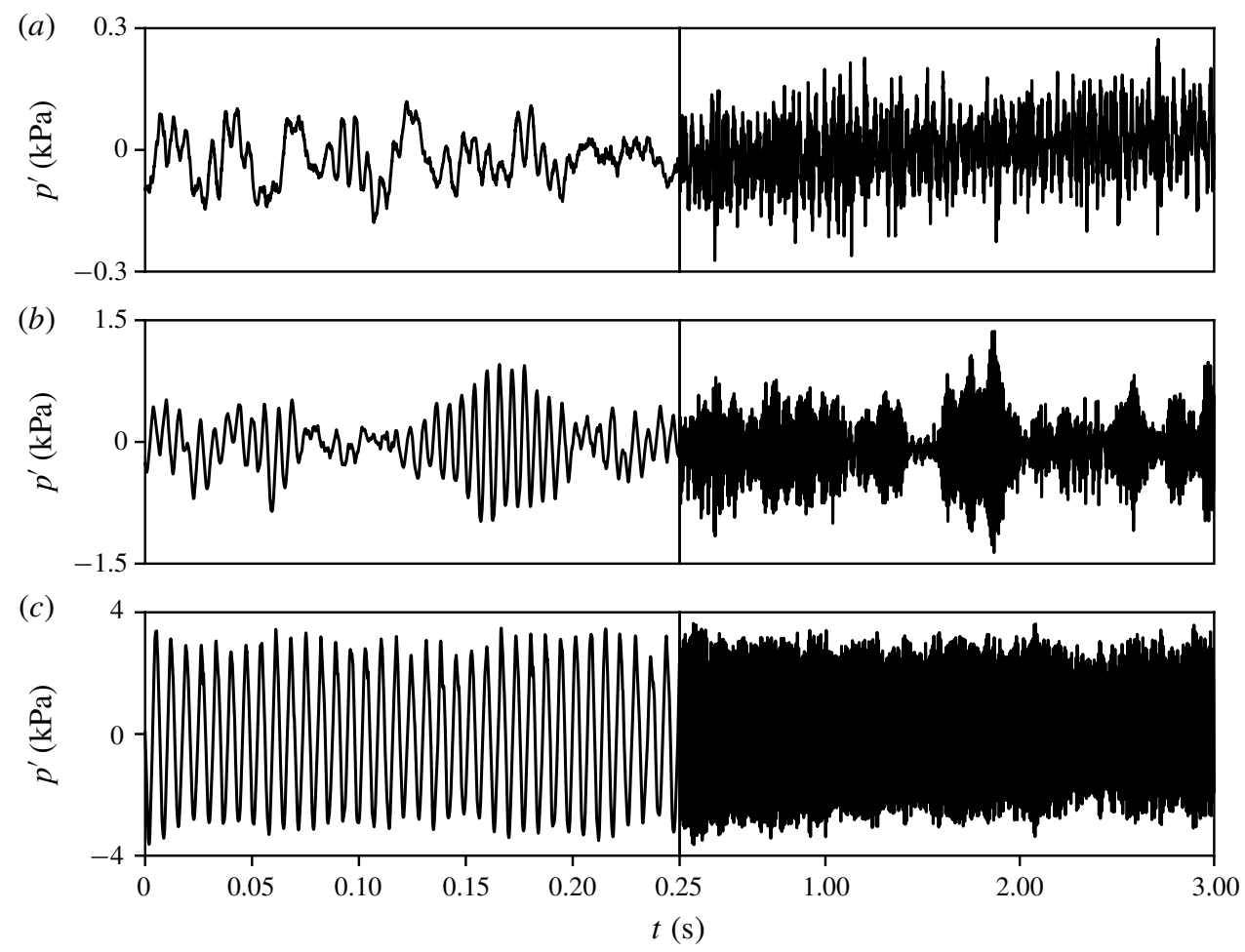

FIGURE 2. The time series of acoustic pressure obtained from the experiments during (a) combustion noise $(\phi=0.97),(b)$ intermittency $(\phi=0.81)$ and $(c)$ thermoacoustic instability $(\phi=0.62)$. During combustion noise, the pressure fluctuations have low amplitude and are aperiodic in nature. During intermittency, we observe high-amplitude bursts of periodic oscillations embedded amidst epochs of low-amplitude aperiodic fluctuations. Thermoacoustic instability is characterized by high-amplitude periodic oscillations with a frequency of $142 \mathrm{~Hz}$. The equivalence ratio is reduced by keeping the fuel flow rate constant and increasing the air flow rate.

are connected if $\omega_{i}, \omega_{j}>0$ or $\omega_{i}, \omega_{j}<0$. Similar to the acoustic power network, here also, we only consider the nearest neighbours $n_{i}$ of a node when establishing a link (the distance between the two nodes should not exceed $\sqrt{ } 2$ ). Hence, a node can have a maximum of only eight connections. The average vorticity $\left(\left(\omega_{i}+\omega_{j}\right) / 2\right)$ at the two connecting nodes is considered as the weight of the link between them. Positive values of node strength denote regions of anticlockwise vorticity $(\omega>0)$ and negative values of node strength denote regions of clockwise vorticity $(\omega<0)$. In this manner, we construct both the acoustic power and the vorticity network at every instant of time for each of the equivalence ratios as the turbulent combustor transits from combustion noise to thermoacoustic instability via intermittency.

\section{Results and discussion}

The thermoacoustic system in a turbulent combustor transits from combustion noise to thermoacoustic instability via a dynamical state called intermittency (Nair et al. 2014). The time trace of pressure fluctuations during the different dynamical states of combustor operation is shown in figure 2 , wherein the equivalence ratio is varied from 
near stoichiometry to lean blowoff conditions. When the equivalence ratio is near stoichiometry $(\phi=0.97)$, the temporal dynamics of the combustor is characterized by low-amplitude aperiodic pressure fluctuations. This stable state of combustor operation is called combustion noise (Strahle 1971). As the equivalence ratio is reduced, the turbulent combustor exhibits intermittency. During intermittency $(\phi=0.81)$, the time series of acoustic pressure consists of bursts of high-amplitude periodic oscillations sandwiched between epochs of low-amplitude aperiodic fluctuations in a seemingly random manner. On further reduction of the equivalence ratio $(\phi=0.62)$, the onset of thermoacoustic instability occurs, which is characterized by high-amplitude periodic pressure oscillations. In the current study, we construct time-varying weighted local acoustic power and vorticity networks during combustion noise, intermittency and thermoacoustic instability. Using the measures from complex network theory, we first examine the spatiotemporal evolution of acoustic power sources visually and then obtain some statistical measures to quantify the dynamics.

\subsection{Spatiotemporal evolution of acoustic power sources during combustion noise and thermoacoustic instability}

We first examine the spatiotemporal evolution of the acoustic power sources during combustion noise (stable combustion) and thermoacoustic instability (unstable combustion). The spatial distributions of the node strengths (3.1) of the acoustic power and the vorticity networks at different time instants during combustion noise are shown in figure 3. In the acoustic power network (figure $3 b$ ), the blue colour represents the local acoustic power sinks. The colourbar indicates the magnitude of node strength, which corresponds to the local acoustic power production in the turbulent reactive flow field. The spatial distribution of the node strength reveals that the acoustic power production happens in small fragmented clusters during combustion noise. The distribution of the local acoustic power sources resembles small islands scattered in an ocean of acoustic power sinks. In the vorticity network (figure $3 c$ ), the node strength gives the average vorticity in the immediate neighbourhood of a node. Velocity vectors obtained from the analysis of PIV are superimposed onto the vorticity network to understand the flow field better. High values of positive node strength (denoted by red regions) indicate the presence of a train of small discrete vortices in the outer shear layer. High values of negative node strength (denoted by blue regions) in the inner shear layer represents the small vortices shed from the tip of the bluff body. The grey colour mask in the subplots (figures $3 b$ and $c$ ) represents the bluff body. The bluff body shape in figures $3(b)$ and $(c)$ looks dissimilar due to the fact that in figure $3(b)$ the nodes are the pixels whereas in figure $3(c)$, the nodes are the interrogation windows used in the analysis of PIV. The size of the interrogation windows is 32 pixels $\times 32$ pixels (Unni et al. 2018). Hence, the area that a node occupies in figure $3(c)$ is bigger than that in figure $3(b)$. Also, in figure $3(c)$, the mask includes the region where laser reflection is high.

In figure 4, the spatial distributions of the node strengths of the acoustic power and the vorticity networks at different time instants during a periodic cycle of thermoacoustic instability are shown. Unlike the situation during combustion noise, the acoustic power production happens now in large clusters during thermoacoustic instability (figure $4 b$ ). The acoustic power production during thermoacoustic instability is around three orders of magnitude higher than that during combustion noise, as indicated by high values of node strength. High values of positive node strength in the vorticity network (at time instants $\mathrm{A}$ and $\mathrm{B}$ in figure $4 c$ ) coincide with the centre 

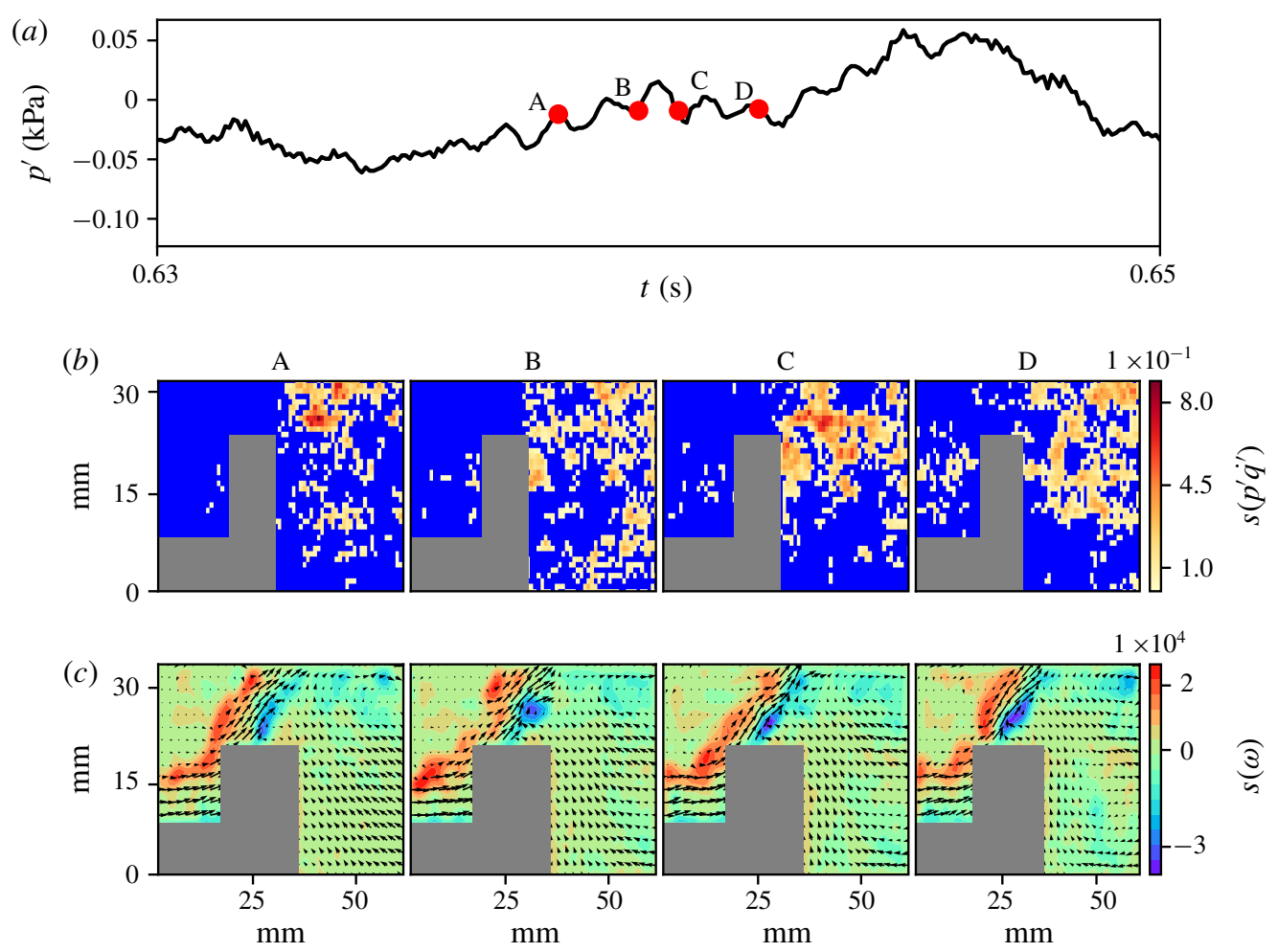

FIgURE 3. (Colour online) The spatial distribution of the node strengths of $(b)$ the acoustic power network and $(c)$ the vorticity network at the corresponding time instants (A, B, C and D) marked on $(a)$ the time series of fluctuating pressure during combustion noise. The blue region in the spatial distribution of the node strength of the acoustic power $\left(p^{\prime} \dot{q}^{\prime}\right)$ network represents local acoustic power sinks. The colourbar in $(b)$ represents the magnitude of the node strength, which indicates the average acoustic power produced in the neighbourhood of a node. The spatial distribution of the node strength shows that acoustic power production happens in small disconnected fragments during combustion noise. In the vorticity network $(c)$, the node strength indicates the average vorticity in the neighbourhood of a node. Positive values of node strength represent the vorticity distribution in the anticlockwise direction, whereas negative node strength indicates the vorticity distribution in the clockwise direction. Velocity vectors are superimposed onto the vorticity network to understand the flow field better. Small vortices are present in the outer shear layer as well, as they are being shed from the tip of the bluff body. The grey rectangles in the subplots represent the bluff body.

of the coherent structure being formed downstream of the bluff body. The coherent structure begins to form at the dump plane, when the acoustic pressure fluctuation is near local minima (E). As the coherent structure convects downstream it grows in size. The formation of large coherent structures at the onset of thermoacoustic instability has been reported in earlier studies, as discussed in $\S 1$. The maximum acoustic power production occurs when the large coherent structure impinges on the top combustor wall.

As the coherent structure convects towards the top of the bluff body (from time instants $\mathrm{A}$ to $\mathrm{C}$ in figure $4 c$ ), the region between the outer and inner shear layer is compressed against the rear end of the bluff body. This increases the shear strain, 

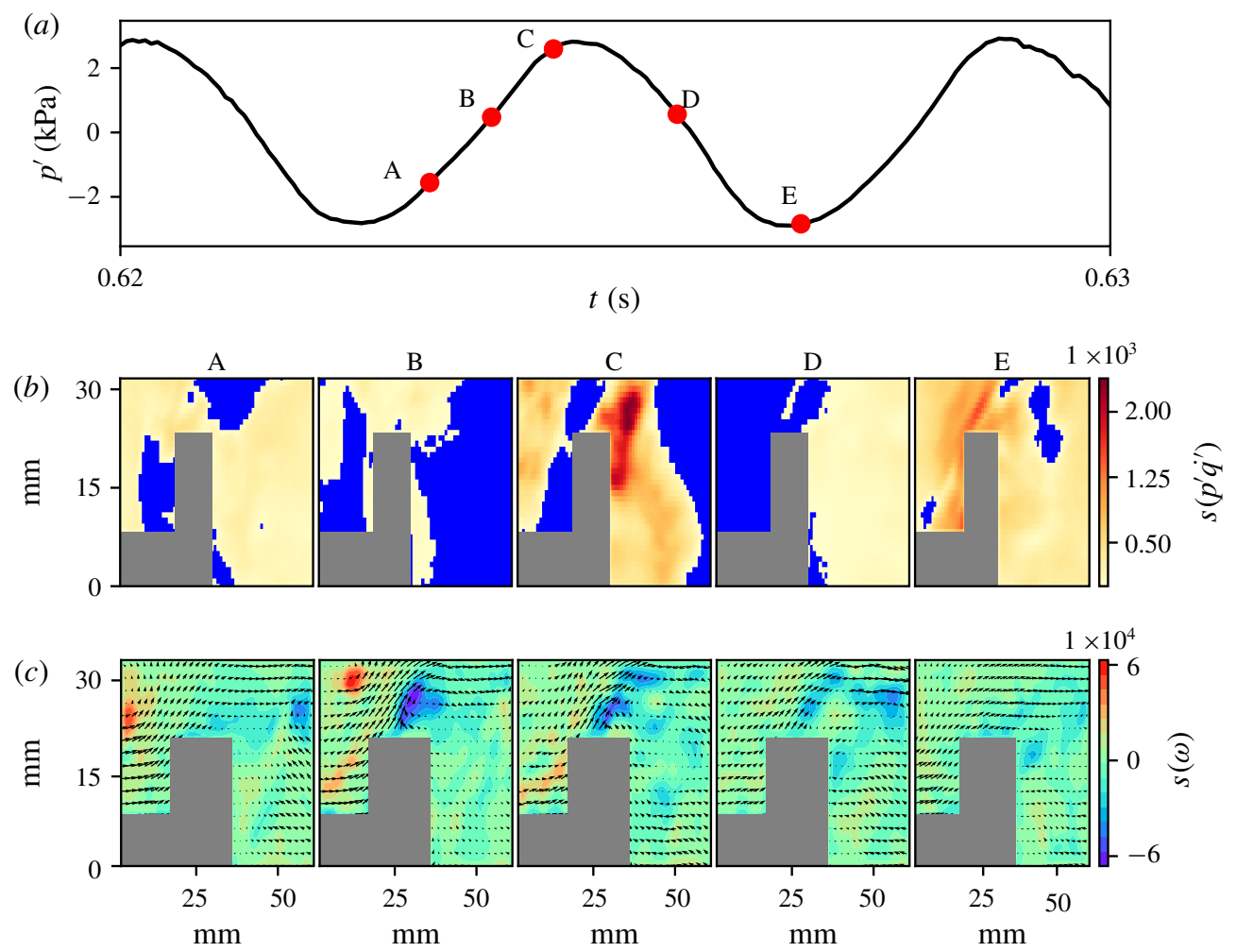

FIgURE 4. (Colour online) The spatial distribution of the node strengths of $(b)$ the acoustic power network and $(c)$ the vorticity network at the corresponding time instants (A, $\mathrm{B}, \mathrm{C}, \mathrm{D}$ and $\mathrm{E}$ ) marked on $(a)$ the time series of oscillating pressure during thermoacoustic instability. The spatial distribution of the node strength of the acoustic power network indicates that during thermoacoustic instability, the acoustic power production occurs in large clusters. The high values of positive node strength of the vorticity network coincide with the centre of the coherent structure formed downstream of the dump plane. The maximum acoustic power production occurs when the coherent structure impinges on the top wall of the combustor $(\mathrm{C})$. The magnitude of acoustic power production during thermoacoustic instability is almost three orders of magnitude higher than that during combustion noise, as indicated by the node strength of the acoustic power network.

which leads to local extinction (Hult et al. 2005). This may be the reason for almost no reaction to take place just upstream of the bluff body, leading to the presence of local acoustic power sinks in the rear end of the bluff body, above the bluff body shaft. As the coherent structure passes over the top of the bluff body, separation of the inner shear layer from the tip of the bluff body happens. This suggests the frequency of formation of the coherent structure downstream of the dump plane and the large vortex from the tip of the bluff body to be almost identical. High values of negative node strength in the vorticity network indicate the shedding of these large vortices from the tip of the bluff body. As the large vortex convects downstream of the bluff body, the region between the outer and the inner shear layer expands. This is accompanied by a flow reversal at the bluff body due to volume expansion because of intense heat release, as well as the reversal of acoustic velocity. This inhibits the flow of fresh reactant mixture into the combustion zone. This is the 
reason why we have large clusters of acoustic power sources in the flow field when the acoustic pressure fluctuation is negative (see time instants $\mathrm{E}$ and $\mathrm{A}$ in figure $4 b$ ). When the pressure inside the combustion chamber becomes very low, the flow surges back into the combustion chamber. As the acoustic mode is reinforced by the intense in-phase heat release rate, the velocity fluctuation amplitude increases. This induces the formation of a large coherent structure downstream of the dump plane and the cycle continues.

\subsection{Spatiotemporal evolution of acoustic power sources during intermittency}

Next, we examine the spatiotemporal evolution of the acoustic power sources during intermittency. Intermittency can be viewed, in simple terms, as a tug of war between the acoustic driving and the acoustic damping present in the system. As the acoustic driving wins over the damping, the growth of the acoustic pressure oscillations occurs. As the damping overtakes the driving, these high-amplitude acoustic pressure oscillations start to decay. It is this competition between the acoustic driving and the damping that leads to the emergence of bursts of high-amplitude periodic oscillations in the background of low-amplitude aperiodic pressure oscillations during intermittency.

In this section, we will see how spatially continuous large clusters of acoustic power sources emerge at the onset of thermoacoustic instability from the small fragmented clusters of acoustic power sources observed during combustion noise. To that end, we investigate the spatial and temporal evolution of acoustic power sources during an aperiodic epoch of intermittency and a periodic epoch of intermittency, the growth of low-amplitude aperiodic pressure fluctuations to high-amplitude periodic oscillations and the decay of high-amplitude periodic pressure oscillations to low-amplitude aperiodic fluctuations. In figure 5, we show the spatiotemporal evolution of acoustic power sources during an aperiodic epoch of intermittency. In this case, similar to the one observed during combustion noise, the acoustic power production happens in small fragmented clusters or islands scattered in an ocean of acoustic power sinks.

Next, we examine the spatiotemporal evolution of acoustic power sources during a periodic epoch of intermittency, as shown in figure 6. We observe coherent structures downstream of the dump plane, as seen during thermoacoustic instability. However, there are a few points to be noted here. Firstly, the size and strength of the coherent structure are not as high as in that observed during thermoacoustic instability. George et al. (2018) quantitatively measured the size of the coherent structures during the different stages of combustor operation using a vortex detection technique. Also, the strength of the coherent structure, which is reflected in the magnitude of the positive node strength, is lower than that during thermoacoustic instability. More significantly, the coherent structure impinges on the rear-end tip of the bluff body (see time instant $\mathrm{C}$ in figure $6 c$ ) and not on the top wall of the combustor. After impingement, the coherent structure breaks down into small vortices. Since the area of impingement of the coherent structure is lower, the heat release rate that follows is not as intense as in the case of thermoacoustic instability. It is to be noted that these coherent structures are not consistently observed during the periodic epochs of intermittency. They are mostly observed during the high-amplitude peaks of the periodic epochs of intermittency. Here also, we observe the shedding of large vortices from the tip of the bluff body. It is to be noted that the size of the clusters of the acoustic power sources is not as large as that observed during thermoacoustic instability.

In order to understand how large clusters of acoustic power sources emerge in the flow field during the periodic epoch of intermittency, we examine the spatiotemporal 

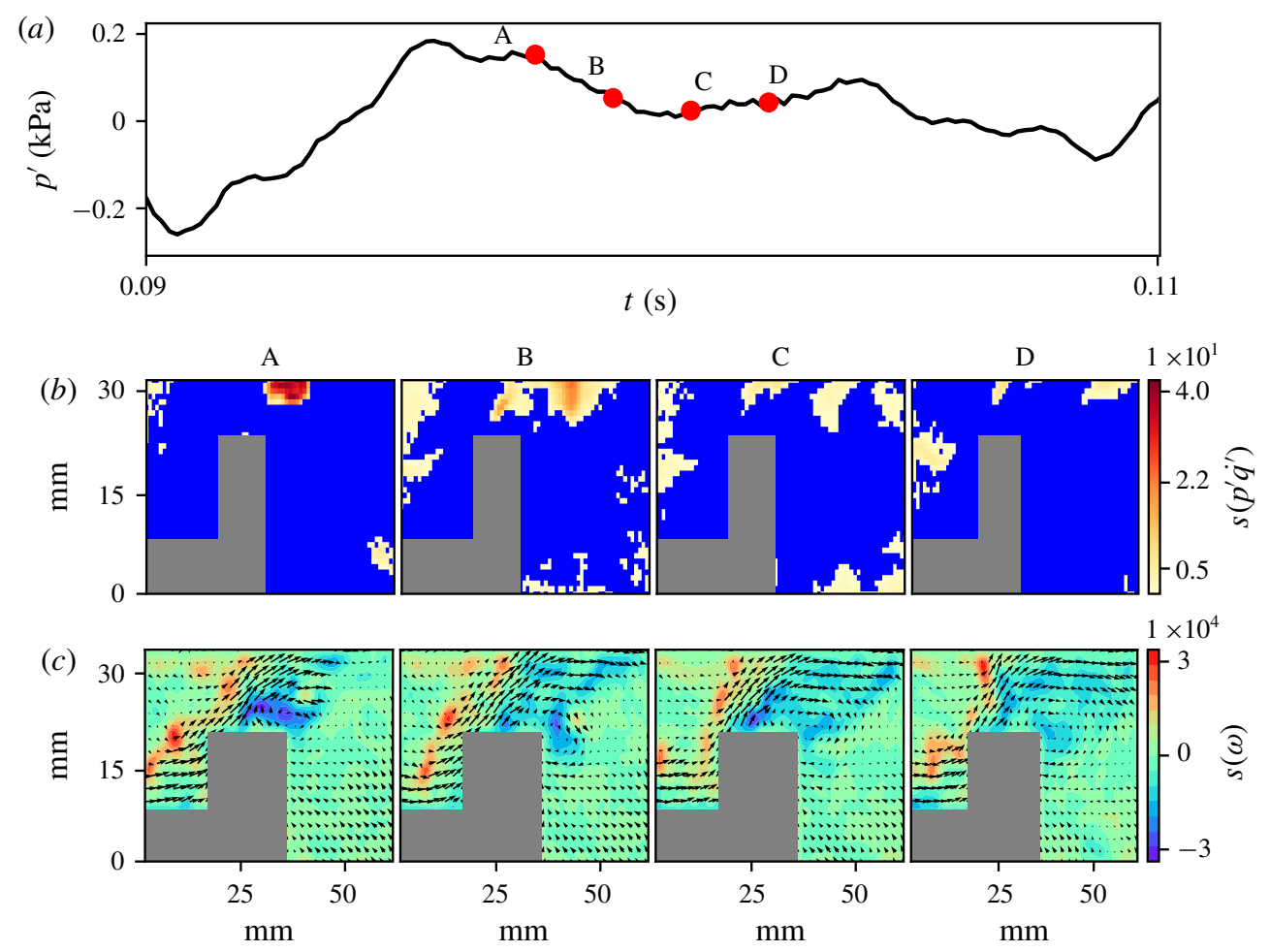

FIgURE 5. (Colour online) The spatial distribution of the node strengths of $(b)$ the acoustic power network and $(c)$ the vorticity network at the corresponding time instants $(\mathrm{A}, \mathrm{B}, \mathrm{C}$ and $\mathrm{D})$ marked on $(a)$ the time series of fluctuating pressure during the aperiodic regime of intermittency. The acoustic power production happens in small fragmented clusters, as in the case of combustion noise.

evolution of the acoustic power sources during the growth of low-amplitude aperiodic fluctuations to high-amplitude periodic oscillations, as shown in figure 7. As the pressure oscillations grow, we find the emergence of acoustic power sources at a greater number of spatial locations in the turbulent reactive flow field. We refer to this phenomenon as the nucleation of acoustic power sources in the flow field. Also, as the acoustic pressure amplitude grows, the small fragmented clusters of acoustic power sources coalesce to form large clusters, as reflected in the spatial distribution of the node strength of the acoustic power network (figure $7 b$ ). The mechanism behind the formation of these large clusters can be deduced by examining the vorticity network (figure $7 c$ ). As the acoustic pressure grows, the magnitude of the positive as well as the negative node strength increases; this denotes the increase in the strength of the vorticity field upstream and downstream of the bluff body, respectively.

During the aperiodic epoch of intermittency, a train of small discrete vortices is observed in the outer shear layer downstream of the dump plane. At the same time, small vortices are shed downstream of the bluff body as the inner shear layer gets separated from the tip of the bluff body. As the perturbations near the dump plane increase, these small vortices in the outer shear layer merge to form large vortices. Merging of vortices leads to an increase in fine scale mixing that accelerates combustion (Schadow \& Gutmark 1992), thus causing the nucleation of acoustic 

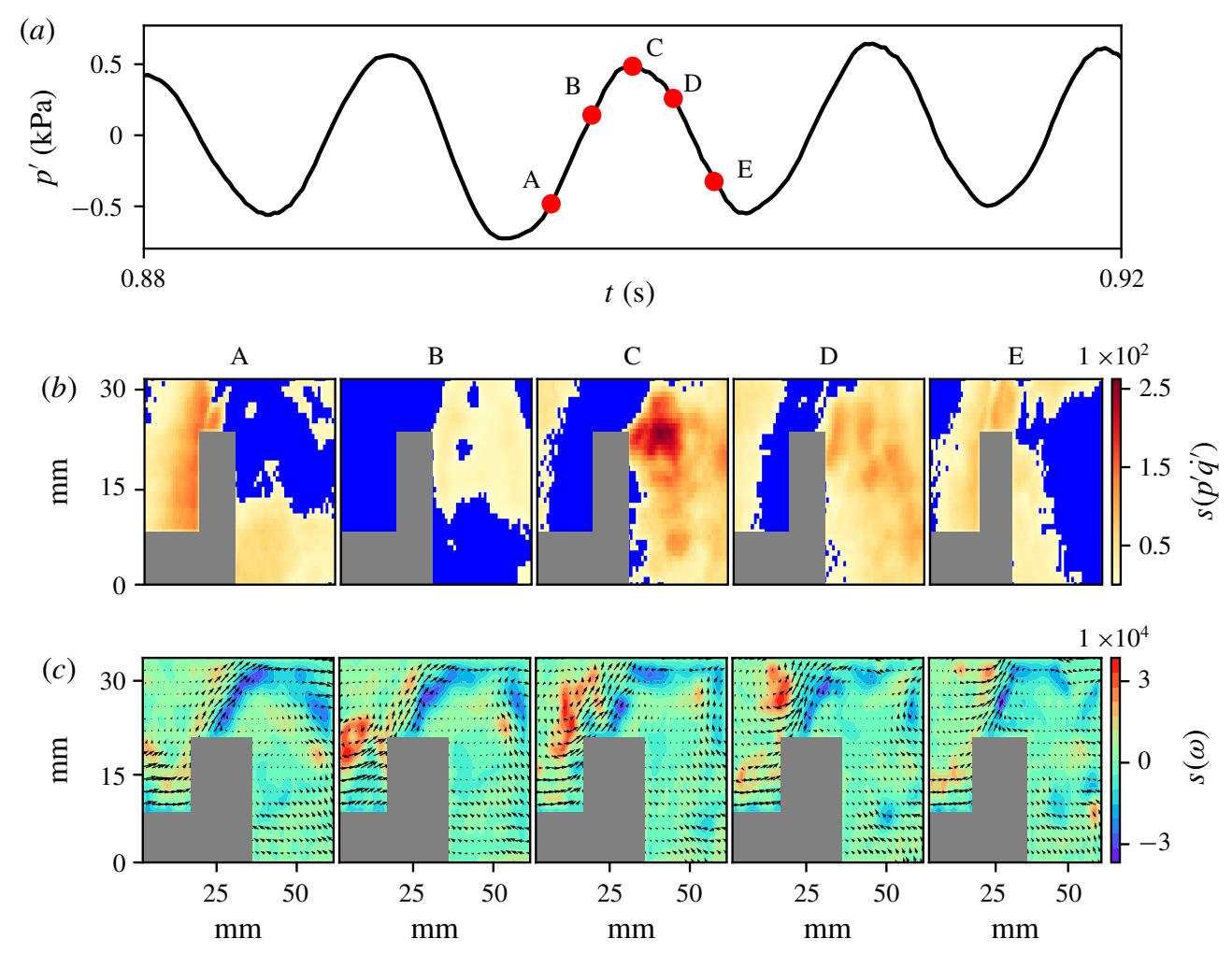

FIgURE 6. (Colour online) The spatial distribution of the node strengths of $(b)$ the acoustic power network and $(c)$ the vorticity network at the corresponding time instants (A, B, C, D and E) marked on $(a)$ the time series of fluctuating pressure during the periodic regime of intermittency. The acoustic power production occurs in large clusters as in the case of thermoacoustic instability. However, unlike the case during thermoacoustic instability, the coherent structure impinges the rear-end tip of the bluff body. After impingement, the coherent structure disintegrates into small vortices that convect over the top of the bluff body.

power sources in the flow field. In addition, as the small vortices merge, coalescence of small fragmented clusters of acoustic power sources occurs. These large vortices convect downstream of the dump plane and impinge on the rear-end tip of the bluff body. Upon impingement, a spike occurs in the heat release rate due to the sudden increased fine scale mixing of the reactants and hot products. This spike in the heat release rate adds energy to the acoustic field, thus acting as a kicked oscillator (Seshadri, Nair \& Sujith 2016). Since the heat release rate occurs in phase with the acoustic pressure oscillation, the amplitude of the acoustic pressure increases, satisfying Rayleigh criteria. This reinforcement of the acoustic mode leads to an increase in the amplitude of velocity fluctuations at the dump plane, thus inducing the formation of a new vortex, and the aforementioned cycle continues. When the perturbation in the flow field is high enough, a large coherent structure forms downstream of the dump plane, gets convected and impinges on the top wall of the combustor. As the large coherent structure carries with it a large pocket of unburnt reactants, upon impingement, the heat release rate is higher. Hence, the energy added to the acoustic field overcomes the damping present in the combustor and the onset 


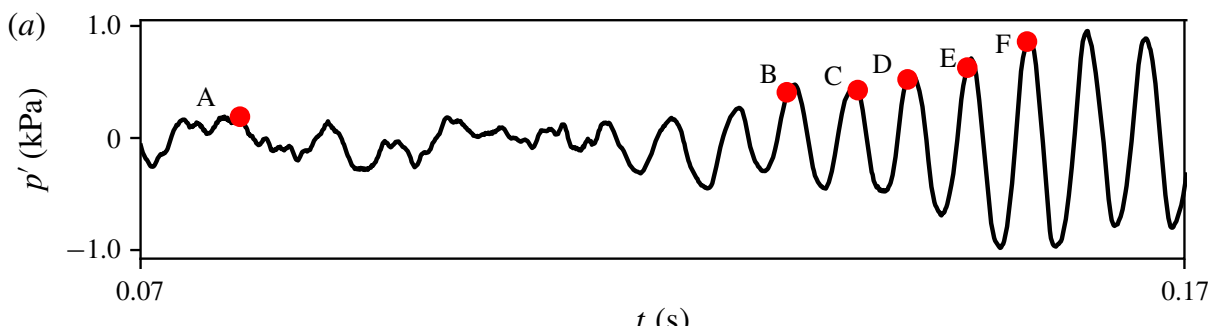

$t(\mathrm{~s})$
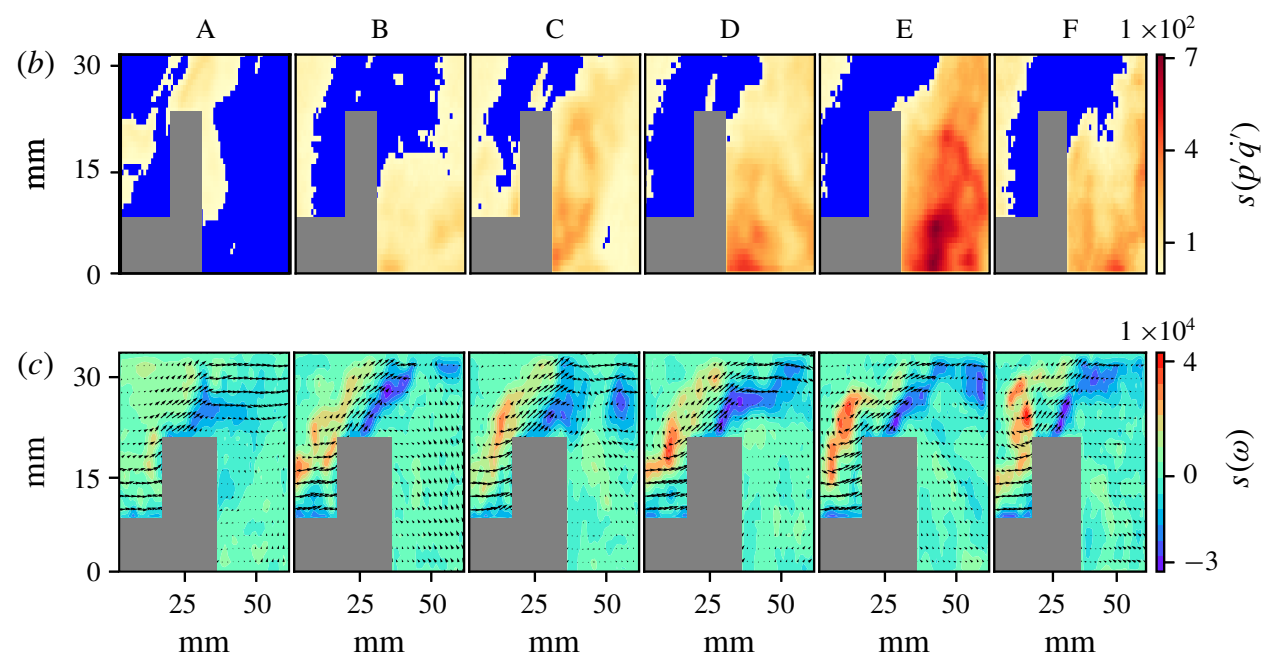

FIgURE 7. (Colour online) The spatial distribution of the node strengths of $(b)$ the acoustic power network and $(c)$ the vorticity network at the corresponding time instants (A, $\mathrm{B}, \mathrm{C}, \mathrm{D}, \mathrm{E}$ and F) marked on $(a)$ the time series of fluctuating pressure during the growth of pressure oscillations observed during intermittency. As the thermoacoustic system transits from low-amplitude aperiodic oscillations to high-amplitude periodic oscillations during intermittency, the acoustic power sources nucleate, coalesce and grow in size and the strength of acoustic power production also increases.

of thermoacoustic instability occurs. We note that the convection of the large vortex towards the rear-end tip of the bluff body/top wall of the combustor perturbs the inner shear layer more and hence the size of the vortices shed downstream of the bluff body also increases.

Finally, we examine the spatiotemporal evolution of acoustic power sources during the decay of high-amplitude periodic pressure oscillations to low-amplitude aperiodic pressure fluctuations, as shown in figure 8. During the high-amplitude periodic pressure oscillations, the acoustic power production occurs in large clusters, as evident at time instant $A$. As the acoustic damping overcomes the acoustic driving in the combustor, the amplitude of the pressure oscillation decays. As the thermoacoustic system transits from high-amplitude periodic oscillations to low-amplitude aperiodic fluctuations, the strength of acoustic power production diminishes and the large clusters disintegrate into small fragmented clusters.

The decay of the acoustic pressure amplitude during intermittency begins to occur once the damping in the turbulent combustor starts to outweigh the acoustic driving. As the damping overcomes the driving, the roll-up of the shear layer into a large 


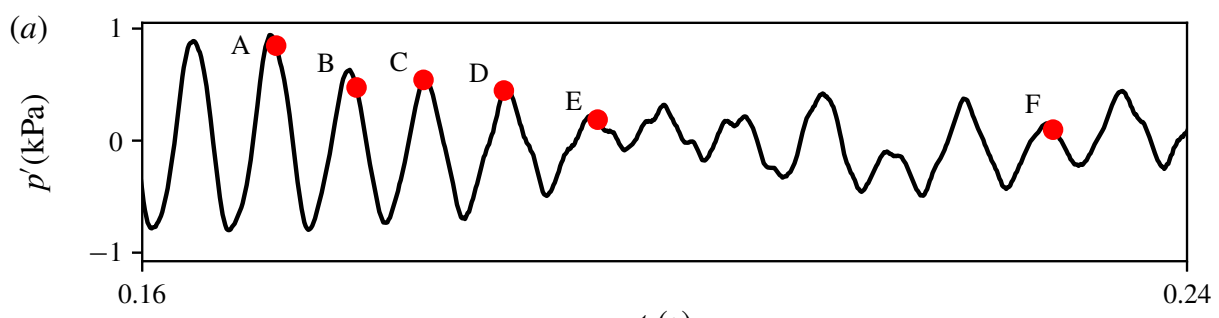

$t(\mathrm{~s})$
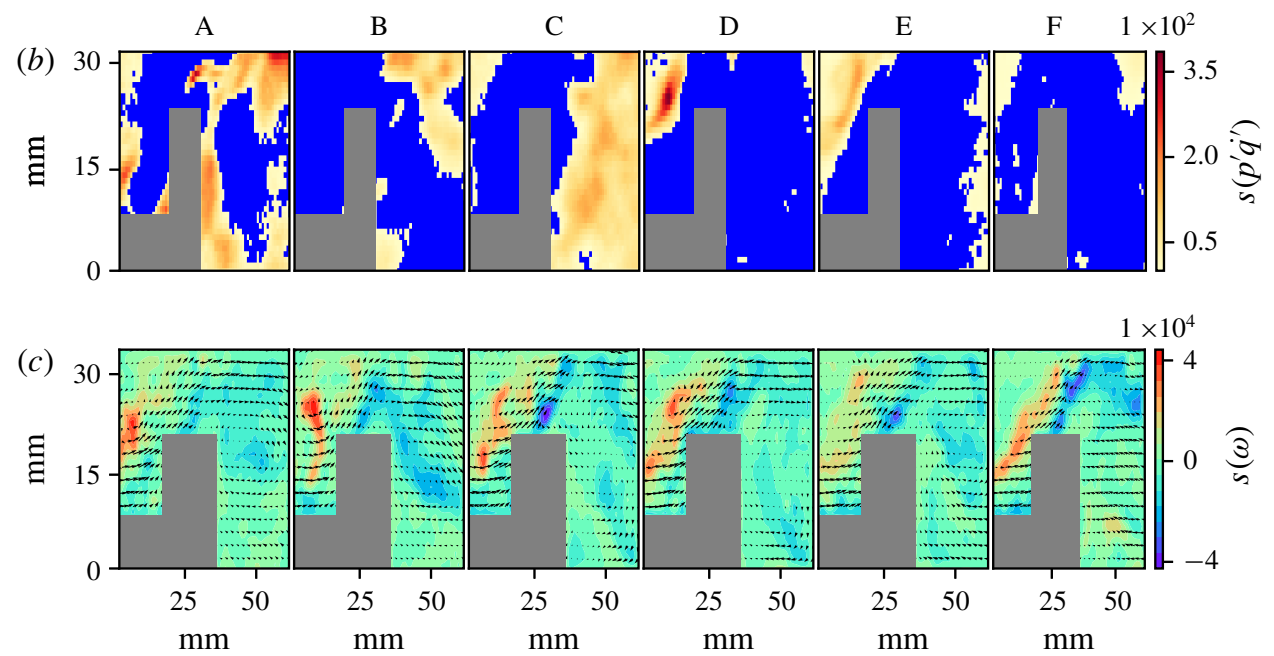

FIGURE 8. (Colour online) The spatial distribution of the node strengths of $(b)$ the acoustic power network and $(c)$ the vorticity network at the corresponding time instants (A, B, C, D, E and F) marked on $(a)$ the time series of fluctuating pressure during the decay of pressure oscillations observed during intermittency.

vortex, observed during the periodic epochs of intermittency, fails to happen, thus ceasing the formation of large clusters of acoustic power sources. Hence, the amount of energy added to the acoustic field reduces. Henceforth, the periodic epochs of intermittency fail to persist and decay, thus preventing the onset of full-blown thermoacoustic instability.

Thus, we observe that the emergence of large clusters of spatially coherent acoustic power sources at the onset of thermoacoustic instability happens via the phenomena of nucleation, coalescence and growth of acoustic power sources. The physical mechanisms behind these phenomena are the merging of small-scale vortices and the formation of larger vortices as the perturbations to the shear layer increase. These findings can be generalized to turbulent thermoacoustic systems involving large-scale vortex shedding. The formation of spatially coherent regions of acoustic power sources at the onset of thermoacoustic instability has also been reported in swirl stabilized combustors (Paschereit, Gutmark \& Weisenstein 1999; Lee \& Santavicca 2003; Huang \& Ratner 2009; Tachibana et al. 2015; Okuno et al. 2015; Kheirkhah et al. 2016; Pawaret al. 2018). Hence, we can hypothesize that the phenomena of nucleation, coalescence and growth of acoustic power sources would be happening during intermittency. However, the physical mechanisms leading to the phenomena of nucleation, coalescence and growth may be different as the flow field in a swirl 
stabilized combustor is different from that observed in a bluff body stabilized combustor.

\subsection{Statistical analysis of the clusters of acoustic power sources}

In this section, we perform a statistical analysis of the clusters of acoustic power sources as the thermoacoustic system transits from a state of combustion noise to thermoacoustic instability via intermittency. The use of complex network theory enables us to quantify the spatiotemporal evolution of the clusters of acoustic power sources. To that end, we calculate the link density $(\rho)$, the size of the giant cluster $(S)$ and the number of clusters $(N)$ at different equivalence ratios as the combustor transits from combustion noise to thermoacoustic instability.

Link density refers to the fraction of the links or edges present in the network. If $n$ represents the total number of nodes and $e$ represents the actual number of links in the network, then link density is given by

$$
\rho=\frac{2 e}{n(n-1)} .
$$

The value of $\rho$ lies between 0 and 1. In simple terms, $\rho$ reflects the number of connections among the nodes of the network. A high value of $\rho$ implies that the network is densely connected and a low value implies that the network is sparsely connected. In the present analysis, a high value of $\rho$ implies that acoustic power production happens in a large number of spatial locations in the flow field as we do not consider acoustic power sinks in the network construction, as explained in $\S 3$.

In graph theory, a connected component of an undirected graph refers to a subgraph in which any two nodes are connected to each other by a path, i.e. a sequence of links (Newman 2010). In the present study, the connected components refer to islands or clusters of acoustic power sources. We track the number of such clusters $(N)$ as well as the size of the giant cluster $(S)$; i.e. the largest connected component of a graph, at each instant of time. In this manner, we obtain, for a given equivalence ratio, $\rho$, $N$ and $S$ at every instant of time. Thus, for each equivalence ratio, we construct 2000 acoustic power networks, corresponding to a duration of $1.0 \mathrm{~s}$, as described in $\S 3$.

Since most of the interesting flow dynamics leading to the coalescence/disintegration of clusters happens upstream of the bluff body, we choose a rectangular ROI upstream of the bluff body for the acoustic power network construction. The chosen ROI, at a distance of $15 \mathrm{~mm}$ downstream of the dump plane and $10 \mathrm{~mm}$ above the bluff body shaft, has physical dimensions of $25 \mathrm{~mm}$ in length and $20 \mathrm{~mm}$ in breadth (figure 9). The ROI has a resolution of 225 pixels $\times 180$ pixels. Since the calculation of network properties with the original resolution would be computationally very intensive, we downsampled the resolution to 112 pixels $\times 90$ pixels $(50 \%$ of the original resolution) using bi-cubic interpolation (Zhang et al. 2011). Hence, the number of nodes in the network reduces from 40500 to 10080 . Also, a small threshold $(\epsilon)$ was introduced for the connections among the nodes to prevent the electronic noise of the high-speed camera, used for chemiluminescence measurements, from creeping into the calculation, especially during combustion noise. We choose the threshold for network link connectivity as $\epsilon=1.0$ (around $5 \%$ of the maximum acoustic power production during combustion noise). Hence, two neighbouring nodes are connected if the values of $p^{\prime} \dot{q}^{\prime}$ at these nodes are greater than 1.0 instead of 0.0 (without any threshold). The sensitivity of the network properties for different thresholds of network link connectivity $(\epsilon)$ and the convergence of the network properties with respect to 


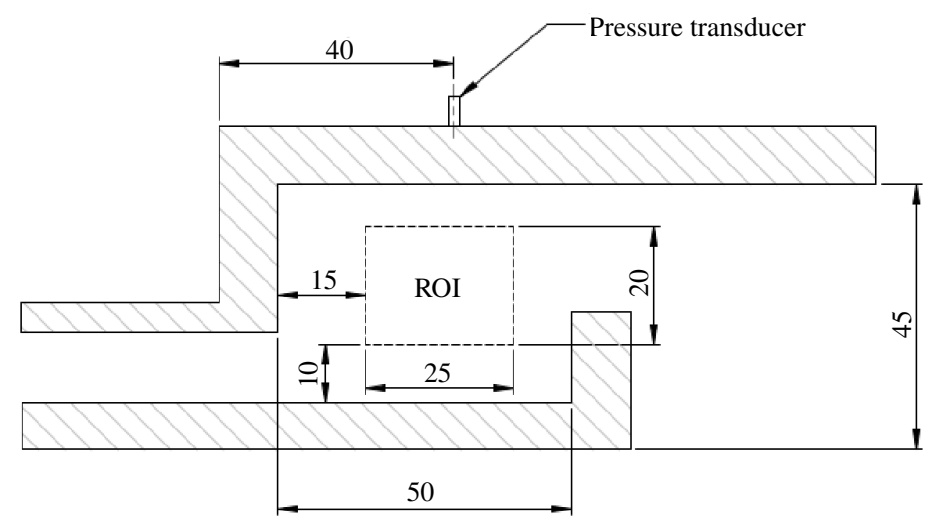

FIGURE 9. Half cross-section of the combustion chamber showing the ROI, in which the acoustic power network is constructed to compute the network properties. The ROI is chosen such that it coincides with the location of formation of coherent structures downstream of the dump plane. The pressure transducer used for the acoustic pressure measurement is located $40 \mathrm{~mm}$ downstream of the dump plane.

the number of images/networks considered for their calculation are discussed in appendix A (see figures 12 and 13). In this study, we use the NetworkX Python package to calculate the network properties (Hagberg, Swart \& Chult 2008).

Figure 10 illustrates the variation of the temporal mean of $\rho$ (figure 10a), $S$ (figure 10b) and $N$ (figure $10 c$ ) of the acoustic power sources at seven different equivalence ratios that cover the transition of the thermoacoustic system from combustion noise to thermoacoustic instability via intermittency. The dashed vertical lines in the plot separate the combustor operational regime into combustion noise, intermittency and thermoacoustic instability, respectively. The variation of the root mean square (r.m.s.) value of the acoustic pressure is also plotted along with the variation of the network properties. As the thermoacoustic system transits from combustion noise to thermoacoustic instability, the average $\rho$ increases sevenfold. This implies that the average number of links during thermoacoustic instability is around seven times that during combustion noise (4.1). A higher value of the average number of links during thermoacoustic instability implies that the acoustic power production occurs at a large number of locations in the flow field when compared to that during combustion noise. This reflects the phenomenon of nucleation of acoustic power sources occurring in the turbulent reactive flow field. The very low value of the average $\rho$ (the maximum value is around 0.0004 ) is due to the fact that only the nearest neighbours of a node are considered when establishing a link, as mentioned in $\$ 3$.

During combustion noise, the acoustic power production happens mostly in a large number of fragmented clusters or islands in an ocean of acoustic power sinks. This explains the presence of a large number of clusters of acoustic power sources during the occurrence of combustion noise in figure 10(c). Mondal et al. (2017) attributed this spatial incoherence in the acoustic power production to the random distribution of instantaneous phase between the pressure oscillations and the heat release rate oscillations during the occurrence of combustion noise. Also, the average $S$ (in figure $10 b$ ) is very small during combustion noise, as the acoustic power production 

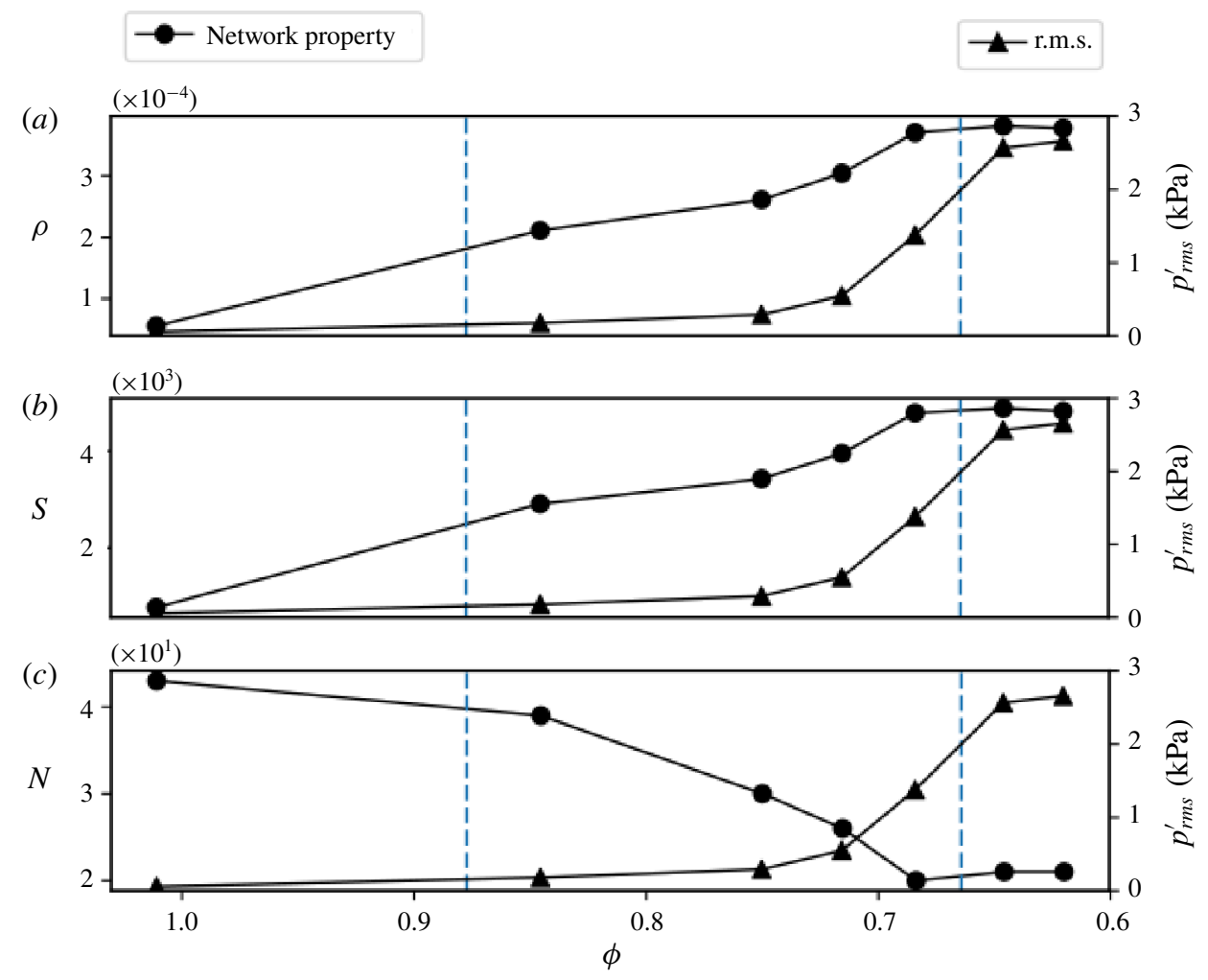

FIGURE 10. (Colour online) Variation of the temporal mean of the network properties: (a) the link density $(\rho)$, (b) the size of the giant cluster $(S)$ and $(c)$ the number of clusters $(N)$, along with the variation of the root mean square (r.m.s.) value of the acoustic pressure as the thermoacoustic system transits from combustion noise to thermoacoustic instability via intermittency. The increase in the link density indirectly indicates the increase in the number of locations in the flow field where acoustic power production occurs. The increase in the size of the giant cluster along with the decrease in the number of clusters show that the acoustic power sources coalesce to form large clusters at the onset of thermoacoustic instability. The dashed vertical lines divide the parameter space, i.e. the equivalence ratio $(\phi)$, into regimes of combustion noise, intermittency and thermoacoustic instability, respectively.

occurs in small fragmented clusters. The average $S$ during thermoacoustic instability is approximately six times the value observed during combustion noise. Additionally, we observe only half the average $N$ at the onset of thermoacoustic instability when compared to that during combustion noise. These observations clearly show that the large number of fragmented clusters of acoustic power sources observed during combustion noise coalesce to form large clusters at the onset of thermoacoustic instability.

Figure 11 shows the variation of the standard deviation of $\rho$ (figure 11a), $S$ (figure $11 b$ ) and $N$ (figure 11c) of the acoustic power sources, along with the variation of the r.m.s. value of the acoustic pressure. During combustion noise, there are instances where large clusters of acoustic power sources are present in the flow field upstream of the bluff body. However, these large clusters having positive values of $p^{\prime} \dot{q}^{\prime}$ occur mostly when the acoustic pressure fluctuation is negative. During 

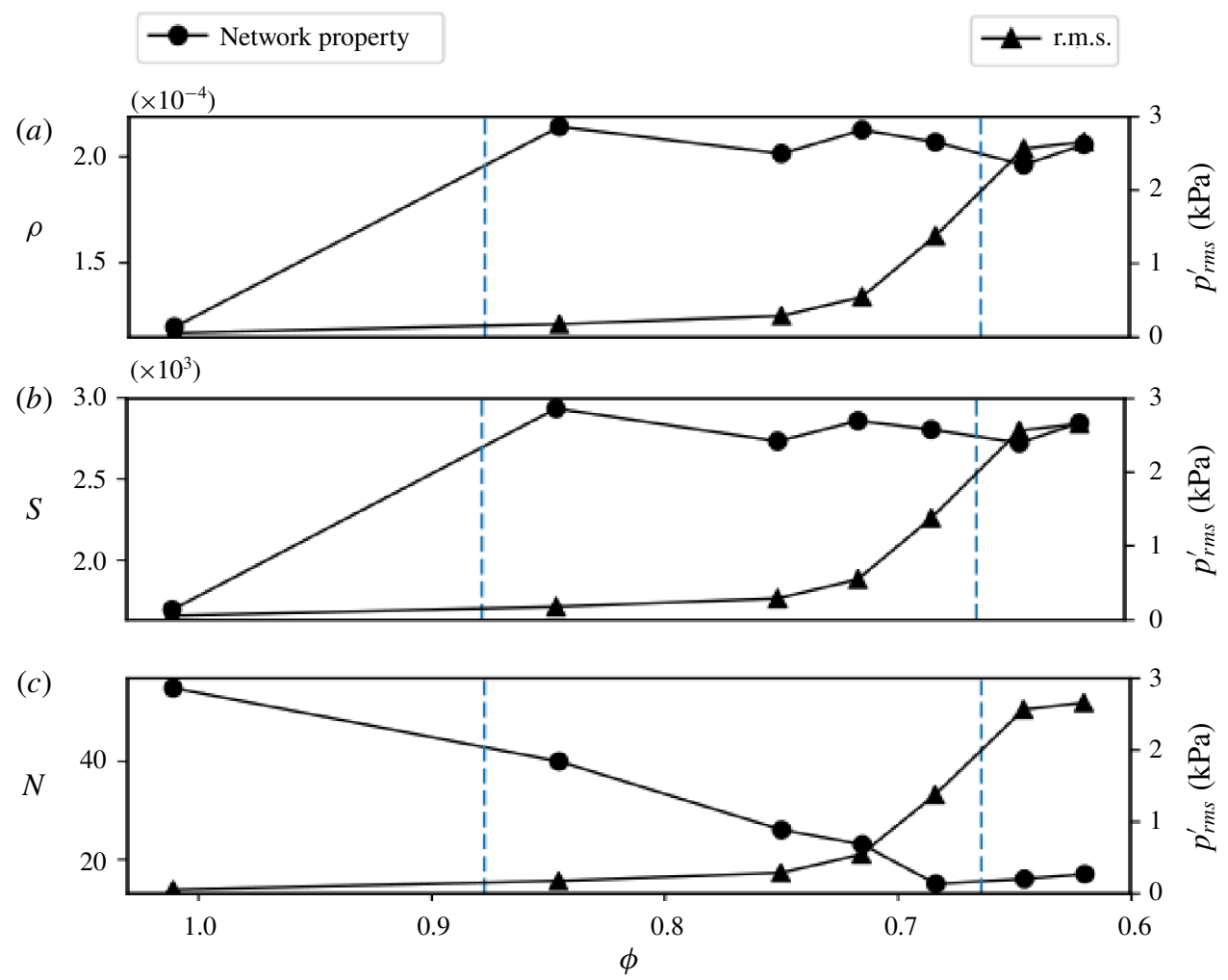

FIGURE 11. (Colour online) Variation of the standard deviation of the network properties: (a) the link density $(\rho),(b)$ the size of the giant cluster $(S)$ and $(c)$ the number of clusters $(N)$, along with the variation of the r.m.s. value of the acoustic pressure as the thermoacoustic system transits from combustion noise to thermoacoustic instability via intermittency. The dashed vertical lines divide the parameter space, i.e. the equivalence ratio $(\phi)$, into regimes of combustion noise, intermittency and thermoacoustic instability, respectively.

combustion noise, most of the reaction occurs in the recirculation zone downstream of the bluff body. In the ROI considered for the calculation of the network properties, which is upstream of the bluff body, not much reaction happens most of the time. Hence, when the acoustic pressure fluctuation $\left(p^{\prime}\right)$ and the local heat release rate fluctuation $\left(\dot{q}^{\prime}\right)$ become negative simultaneously, we observe large clusters of acoustic power sources (positive values of $p^{\prime} \dot{q}^{\prime}$ ) upstream of the bluff body. This explains the high value of the standard deviation for $\rho, S$ and $N$ during combustion noise. However, we observe high values of standard deviation only for $\rho$ and $S$ during intermittency. This can be attributed to the fact that we observe small fragmented clusters of acoustic power sources during the aperiodic epochs of intermittency and large clusters of acoustic power sources during the periodic epochs of intermittency. The standard deviation for the number of clusters of acoustic power sources is low during thermoacoustic instability compared to that during combustion noise (figure 11c). As discussed earlier, during thermoacoustic instability, the acoustic power production happens mostly in large clusters. Hence, the average number of clusters (see figure 10c) as well as the standard deviation (figure 11c) is low during 
thermoacoustic instability. During one acoustic cycle, when the coherent structure convects out of the ROI towards the top of the bluff body, there will not be many acoustic power sources inside the region of interest. This is the reason for the high value of the standard deviation for link density (figure 11a) and the size of the giant cluster (figure $11 b$ ) during thermoacoustic instability.

We note that the gradual change in the average network properties happens much before the rise in the r.m.s. of the acoustic pressure as the thermoacoustic system transits from combustion noise to thermoacoustic instability via intermittency (figure 10). This suggests that the transition from small fragmented clusters to large clusters of acoustic power sources is not abrupt. In statistical physics, percolation theory is used in lattice models to study the phenomenon of phase transition. The variation of the size of the giant cluster is used as a marker to study the critical behaviour near the percolation threshold (Newman 2010; Barabási 2016). Using complex network theory, researchers have reported percolation-like phase transitions in brain networks (Gallos, Sigman \& Makse 2012; Fornito, Zalesky \& Bullmore 2016), protein networks (Deb, Vishveshwara \& Vishveshwara 2009; Weber \& Pande 2015) and recently in climate networks ( $\mathrm{Lu}$, Yuan \& Fu 2016). In the present study, the variation of the average size of the giant cluster (see figure 10b) reveals a continuous percolation-like phase transition at the onset of thermoacoustic instability in a turbulent combustor. However, a detailed analysis is required to confirm whether this is the case in turbulent combustors with other flame stabilization mechanisms.

\section{Conclusions}

We used time-varying spatial network analysis of the local acoustic power and the vorticity field in a turbulent combustor with a bluff body stabilized flame during the transition from combustion noise to thermoacoustic instability via intermittency. We find that during combustion noise the acoustic power production occurs in small fragmented clusters, whereas during thermoacoustic instability the acoustic power production happens coherently in large areas. This spatial emergence of large clusters of acoustic power production occurs via nucleation, coalescence and growth of acoustic power sources as the acoustic pressure grows during intermittency. As the acoustic pressure decays, the large clusters of acoustic power sources disintegrate into small fragmented clusters. The presence of large clusters of acoustic power sources during the periodic epochs of intermittency persuades the authors to hypothesize that the disruption of these large clusters can prevent an impending thermoacoustic instability. We quantify the spatiotemporal evolution of the acoustic power sources using network measures such as the link density, the size of the giant cluster and the number of clusters of acoustic power sources. An increase in the link density as well as the size of the giant cluster, along with a decrease in the number of clusters, corroborate our findings. A continuous increase in the size of the giant cluster suggests that the onset of thermoacoustic instability in a turbulent combustor can be viewed as a percolation-like phase transition. The approach presented here can be utilized to study the spatiotemporal evolution of acoustic power sources in combustors with other flame stabilization mechanisms.

\section{Acknowledgements}

We acknowledge funding from Siemens Corporate Technology, India, and Office of Naval Research Global (contract monitor Dr R. Kolar). We acknowledge Dr T. Komarek and Professor W. Polifke, TU Munich, Germany, for sharing the design of 
the combustor. We gratefully thank Dr V. R. Unni and V. Godavarthi for their valuable comments and suggestions that helped in improving the quality of the paper. We also acknowledge Mr N. B. George, Mr R. Manikandan and Mr S. Thilagaraj for help in performing the experiments.

\section{Appendix A}

A.1. Sensitivity of network properties with respect to the threshold of network link connectivity $(\epsilon)$

Figure 12 shows the variation of the temporal mean of the network properties, namely $\rho$ (figure 12a), $S$ (figure 12b) and $N$ (figure 12c), with respect to different thresholds of network link connectivity $(\epsilon)$ for a case of combustion noise $(\phi=1.01)$, intermittency $(\phi=0.72)$ and thermoacoustic instability $(\phi=0.64)$. The temporal mean is calculated over 2000 images/networks. The average link density and the average size of the giant cluster remain almost a constant for combustion noise, intermittency and thermoacoustic instability for different thresholds of network link

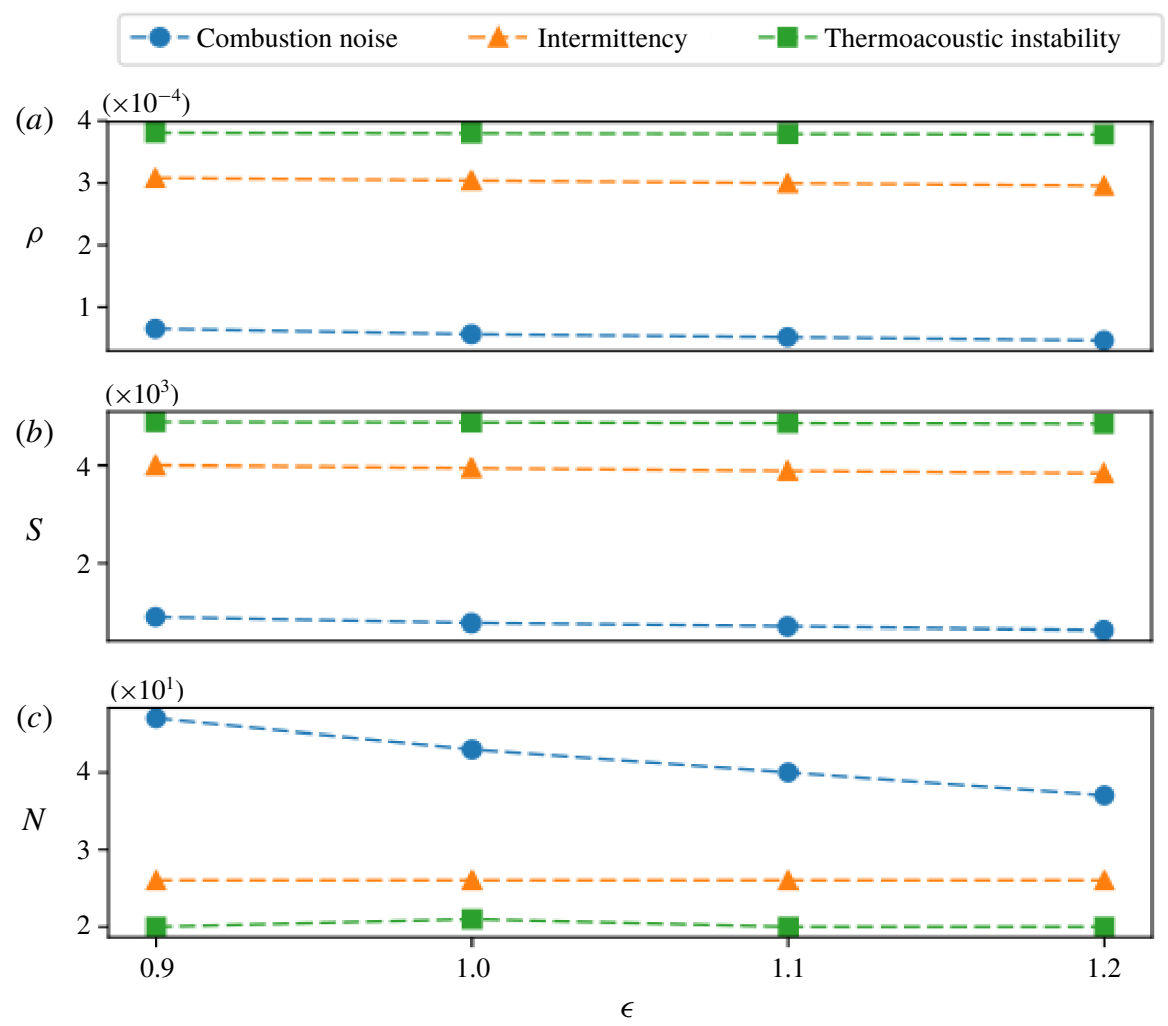

FIGURE 12. (Colour online) Variation of the temporal mean of the network properties: (a) the link density $(\rho)$, (b) the size of the giant cluster $(S)$ and $(c)$ the number of clusters $(N)$ with respect to different thresholds of network link connectivity for a case of combustion noise $(\phi=1.01)$, intermittency $(\phi=0.72)$ and thermoacoustic instability $(\phi=0.64)$. Here, the temporal mean is calculated over 2000 images/networks. We observe that the variation in the temporal mean of the network properties for a threshold of 1.0 and above is negligible. 

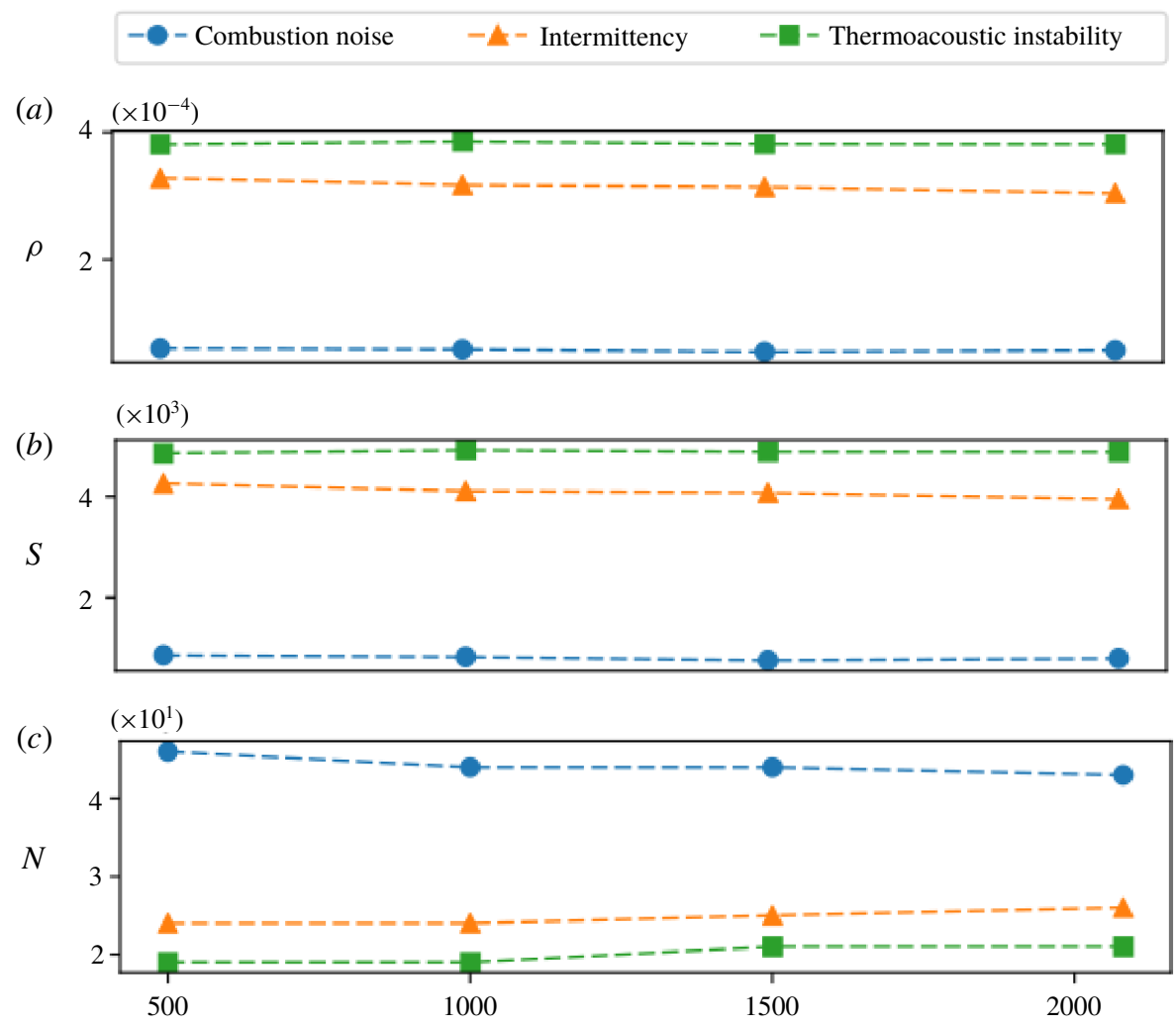

No. of images

FIGURE 13. (Colour online) Variation of the temporal mean of the network properties: (a) the link density $(\rho),(b)$ the size of the giant cluster $(S)$ and $(c)$ the number of clusters $(N)$ calculated over 500, 1000, 1500 and 2080 images/networks for a case of combustion noise $(\phi=1.01)$, intermittency $(\phi=0.72)$ and thermoacoustic instability $(\phi=0.64)$. Here, the threshold for the connectivity of links in the acoustic power network is fixed at 1.0. We observe that the temporal mean of all the network properties for combustion noise, intermittency and thermoacoustic instability remains almost a constant when computed over a total of 1500 images/networks and above.

connectivity. For the case of combustion noise, we observe a decrease of around $7 \%$ and $7.5 \%$ in the average number of clusters of acoustic power sources when $\epsilon$ is increased from 1.0 to 1.1 and from 1.1 to 1.2 , respectively. Nevertheless, the average number of clusters of acoustic power sources does not change for intermittency and thermoacoustic instability for $\epsilon \geqslant 1.0$. This justifies the use of $\epsilon=1.0$ for the network link connectivity in the current analysis.

\section{A.2. Convergence of network properties with respect to the number of images/networks}

Figure 13 shows the variation of the temporal mean of the network properties, namely $\rho$ (figure 13a), $S$ (figure 13b) and $N$ (figure 13c), calculated over different numbers of images/networks for a case of combustion noise $(\phi=1.01)$, intermittency $(\phi=0.72)$ and thermoacoustic instability $(\phi=0.64)$. The average link density 
and the size of the giant cluster remain nearly a constant for combustion noise, intermittency and thermoacoustic instability when calculated over the entire range of number of images/networks. The average number of clusters of acoustic power sources saturates for combustion noise, intermittency and thermoacoustic instability when computed over 1500 images/networks and above. This justifies the choice of 2000 images/networks for the computation of the statistical measures of the network properties.

\section{REFERENCES}

Albert, R. \& BarabÁsi, A. L. 2002 Statistical mechanics of complex networks. Rev. Mod. Phys. 74 (1), 47-97.

Ananthroishnan, N., Deo, S. \& Culick, F. E. 2005 Reduced-order modeling and dynamics of nonlinear acoustic waves in a combustion chamber. Combust. Sci. Technol. 177 (2), 221-248.

Barabasi, A. L. 2003 Linked: How Everything is Connected to Everything Else and What It Means for Business, Science, and Everyday Life. Basic Books.

BarabÁSI, A. L. 2011 The network takeover. Nat. Phys. 8 (1), 14-16.

BARABÁSI, A. L. 2016 Network Science. Cambridge University Press.

BARABÁsi, A. L. \& Albert, R. 1999 Emergence of scaling in random networks. Science 286 (5439), 509-512.

Barthélemy, M. 2011 Spatial networks. Phys. Rep. 499 (1-3), 1-101.

BAR-YAM, Y. 1997 Dynamics of Complex Systems, vol. 213. Addison-Wesley.

Boccaletti, S., Latora, V., Moreno, Y., Chavez, M. \& Hwang, D. U. 2006 Complex networks: structure and dynamics. Phys. Rep. 424 (4-5), 175-308.

Cabral, J., Kringelbach, M. L. \& Deco, G. 2017 Functional connectivity dynamically evolves on multiple time-scales over a static structural connectome: models and mechanisms. NeuroImage 160, 84-96.

CAndel, S. 2002 Combustion dynamics and control: progress and challenges. Proc. Combust. Inst. 29 (1), 1-28.

Clavin, P., Kim, J. S. \& Williams, F. A. 1994 Turbulence-induced noise effects on high-frequency combustion instabilities. Combust. Sci. Technol. 96 (1-3), 61-84.

Coats, C. M. 1996 Coherent structures in combustion. Prog. Energy Combust. Sci. 22 (5), 427-509.

Deb, D., Vishveshwara, S. \& Vishveshwara, S. 2009 Understanding protein structure from a percolation perspective. Biophys. J. 97 (6), 1787-1794.

Dimitriadis, S. I., Laskaris, N. A., Tsirka, V., Vourkas, M., Micheloyannis, S. \& Fotopoulos, S. 2010 Tracking brain dynamics via time-dependent network analysis. J. Neurosci. Meth. 193 (1), 145-155.

Donges, J. F., Zou, Y., Marwan, N.\& Kurths, J. 2009 Complex networks in climate dynamics. Eur. Phys. J. 174 (1), 157-179.

Doron, K. W., Bassett, D. S. \& Gazzaniga, M. S. 2012 Dynamic network structure of interhemispheric coordination. Proc. Natl Acad. Sci. USA 109 (46), 18661-18668.

Emerson, B., O'Connor, J., Juniper, M. \& Lieuwen, T. 2012 Density ratio effects on reacting bluff-body flow field characteristics. J. Fluid Mech. 706, 219-250.

Fornito, A., Zalesky, A. \& Bullmore, E. 2016 Fundamentals of Brain Network Analysis. Academic Press.

Gallos, L., Sigman, M. \& Makse, H. 2012 The conundrum of functional brain networks: smallworld efficiency or fractal modularity. Front. Physiol. 3, 123.

George, N. B., Unni, V. R., Raghunathan, M. \& Sujith, R. I. 2018 Pattern formation during transition from combustion noise to thermoacoustic instability via intermittency. J. Fluid Mech. 849, 615-644.

Godavarthi, V., Unni, V. R., Gopalakrishnan, E. A. \& Sujith, R. I. 2017 Recurrence networks to study dynamical transitions in a turbulent combustor. Chaos 27 (6), 063113. 
Gotoda, H., Amano, M., Miyano, T., Ikawa, T., Maki, K. \& Tachibana, S. 2012 Characterization of complexities in combustion instability in a lean premixed gas-turbine model combustor. Chaos 22 (4), 043128.

Gotoda, H., Kinugawa, H., Tsujimoto, R., Domen, S. \& Okuno, Y. 2017 Characterization of combustion dynamics, detection, and prevention of an unstable combustion state based on a complex-network theory. Phys. Rev. Appl. 7 (4), 044027.

Gotoda, H., Nikimoto, H., Miyano, T. \& Tachibana, S. 2011 Dynamic properties of combustion instability in a lean premixed gas-turbine combustor. Chaos 21 (1), 013124.

Gotoda, H., Shinoda, Y., Kobayashi, M., OKuno, Y. \& Tachibana, S. 2014 Detection and control of combustion instability based on the concept of dynamical system theory. Phys. Rev. E 89 (2), 022910.

Hagberg, A. A., Schult, D. A. \& Swart, P. J. 2008 Exploring network structure, dynamics, and function using NetworkX. In Proceedings of the 7th Python in Science Conference (SciPy2008), Pasadena, CA, USA (ed. G. Varoquaux, T. Vaught \& J. Millman), pp. 11-15.

Hegde, U. G., Reuter, D. \& Zinn, B. T. 1988 Sound generation by ducted flames. AIAA J. 26 (5), 532-537.

Holme, P. \& Saramäki, J. 2012 Temporal networks. Phys. Rep. 519 (3), 97-125.

HUANG, Y. \& RATneR, A. 2009 Experimental investigation of thermoacoustic coupling for low-swirl lean premixed flames. J. Propul. Power 25 (2), 365-373.

Hult, J., Meier, U., Meier, W., Harvey, A. \& Kaminski, C. F. 2005 Experimental analysis of local flame extinction in a turbulent jet diffusion flame by high repetition 2-D laser techniques and multi-scalar measurements. Proc. Combust. Inst. 30 (1), 701-709.

Hurle, I. R., Price, R. B., Sugden, T. M. \& Thomas, A. 1968 Sound emission from open turbulent premixed flames. Proc. R. Soc. Lond. A 303 (1475), 409-427.

JUNiPER, M. P. \& SUJith, R. I. 2018 Sensitivity and nonlinearity of thermoacoustic oscillations. Annu. Rev. Fluid Mech. 50, 661-689.

Kheirkhah, S., Cirtwill, J. M., Saini, P., Venkatesan, K. \& Steinberg, A. M. 2017 Dynamics and mechanisms of pressure, heat release rate, and fuel spray coupling during intermittent thermoacoustic oscillations in a model aeronautical combustor at elevated pressure. Combust. Flame 185, 319-334.

Kheirkhah, S., Geraedts, B. D., Saini, P., Venkatesan, K. \& Steinberg, A. M. 2016 Non-stationary local thermoacoustic phase relationships in a gas turbine combustor. Proc. Combust. Inst. 36 (3), 3873-3880.

Komarek, T. \& PolifKe, W. 2010 Impact of swirl fluctuations on the flame response of a perfectly premixed swirl burner. Trans. ASME J. Engng Gas Turbines Power 132 (6), 061503.

Lacasa, L., Luque, B., Ballesteros, F., Luque, J. \& Nuno, J. C. 2008 From time series to complex networks: the visibility graph. Proc. Natl Acad. Sci. USA 105 (13), 4972-4975.

LeE, J. G. \& SAntavicCA, D. A. 2003 Experimental diagnostics for the study of combustion instabilities in lean premixed combustors. J. Propul. Power 19 (5), 735-750.

Lieuwen, T. C. 2002 Experimental investigation of limit-cycle oscillations in an unstable gas turbine combustor. J. Propul. Power 18 (1), 61-67.

Lieuwen, T. C. \& Banaszuk, A. 2005 Background noise effects on combustor stability. J. Propul. Power 21 (1), 25-31.

LU, Z., YUAN, N. \& FU, Z. 2016 Percolation phase transition of surface air temperature networks under attacks of El Niño/La Niña. Sci. Rep. 6, 26779.

Malik, N., Bookhagen, B., Marwan, N. \& Kurths, J. 2012 Analysis of spatial and temporal extreme monsoonal rainfall over South Asia using complex networks. Climate Dyn. 39 (3-4), 971-987.

Mcmanus, K. R., Poinsot, T. \& Candel, S. M. 1993 A review of active control of combustion instabilities. Prog. Energy Combust. Sci. 19 (1), 1-29.

Molkenthin, N., Rehfeld, K., Marwan, N. \& Kurths, J. 2014 Networks from flows - from dynamics to topology. Sci. Rep. 4, 4119.

Mondal, S., Unni, V. R. \& SUJith, R. I. 2017 Onset of thermoacoustic instability in turbulent combustors: an emergence of synchronized periodicity through formation of chimera-like states. J. Fluid Mech. 811, 659-681. 
Murugesan, M. \& Sujith, R. I. 2015 Combustion noise is scale-free: transition from scale-free to order at the onset of thermoacoustic instability. J. Fluid Mech. 772, 225-245.

Nair, V., Thampi, G., Karuppusamy, S., Gopalan, S. \& Sujith, R. I. 2013 Loss of chaos in combustion noise as a precursor of impending combustion instability. Intl J. Spray Combust. Dyn. 5 (4), 273-290.

NAIR, V., Thampi, G. \& SUJith, R. I. 2014 Intermittency route to thermoacoustic instability in turbulent combustors. J. Fluid Mech. 756, 470-487.

Newman, M. 2010 Networks. Oxford University Press.

Nicosia, V., Tang, J., Musolesi, M., Russo, G., Mascolo, C. \& Latora, V. 2012 Components in time-varying graphs. Chaos 22 (2), 023101.

Noiray, N. \& Schuermans, B. 2013a Deterministic quantities characterizing noise driven Hopf bifurcations in gas turbine combustors. Intl J. Non-Linear Mech. 50, 152-163.

Noiray, N. \& SChuermans, B. $2013 b$ On the dynamic nature of azimuthal thermoacoustic modes in annular gas turbine combustion chambers. Proc. R. Soc. Lond. A 469 (2151), 20120535.

Nori, V. \& SeItzman, J. 2008 Evaluation of chemiluminescence as a combustion diagnostic under varying operating conditions. In 46th AIAA Aerospace Sciences Meeting and Exhibit, p. 953.

OKuno, Y., Small, M. \& Gotoda, H. 2015 Dynamics of self-excited thermoacoustic instability in a combustion system: pseudo-periodic and high-dimensional nature. Chaos 25 (4), 043107.

Paschereit, C. O., Gutmark, E. \& Weisenstein, W. 1999 Coherent structures in swirling flows and their role in acoustic combustion control. Phys. Fluids 11 (9), 2667-2678.

Pawar, S. A., Mondal, S., George, N. B. \& Sujith, R. I. 2018 Synchronization behaviour during the dynamical transition in swirl-stabilized combustor: temporal and spatiotemporal analysis. In 2018 AIAA Aerospace Sciences Meeting, p. 0394.

Pawar, S. A., Seshadri, A., UnNi, V. R. \& Sujith, R. I. 2017 Thermoacoustic instability as mutual synchronization between the acoustic field of the confinement and turbulent reactive flow. J. Fluid Mech. 827, 664-693.

Pawar, S. A.\& Sujith, R. I. 2018 Transition to thermoacoustic instability in a turbulent combustor. J. Combust. Soc. Japan 60 (192), 99-111.

Poinsot, T. J., Trouve, A. C., Veynante, D. P., Candel, S. M. \& Esposito, E. J. 1987 Vortex-driven acoustically coupled combustion instabilities. J. Fluid Mech. 177, 265-292.

Poinsot, T. \& Veynante, D. 2005 Theoretical and Numerical Combustion. RT Edwards, Inc.

Putnam, A. A. 1971 Combustion Driven Oscillations in Industry. Elsevier.

Raffel, M., Willert, C.E. \& Kompenhans, J. 2007 Particle Image Velocimetry: A Practical Guide. Springer.

RaYleigh, J. W. S. 1878 The explanation of certain acoustical phenomena. Nature 18 (455), 319-321.

Rogers, D. E. \& MARble, F. E. 1956 A mechanism for high-frequency oscillation in ramjet combustors and afterburners. J. Jet Propul. 26 (6), 456-462.

Scarsoglio, S., Iacobello, G. \& Ridolfi, L. 2016 Complex networks unveiling spatial patterns in turbulence. Intl J. Bifurcation Chaos 26 (13), 1650223.

Schadow, K. C. \& Gutmark, E. 1992 Combustion instability related to vortex shedding in dump combustors and their passive control. Prog. Energy Combust. Sci. 18 (2), 117-132.

Schwing, J., Sattelmayer, T. \& Noiray, N. 2011 Interaction of vortex shedding and transverse high-frequency pressure oscillations in a tubular combustion chamber. In ASME 2011 Turbo Expo: Turbine Technical Conference and Exposition, pp. 259-268. American Society of Mechanical Engineers.

Seshadri, A., NAir, V. \& Sujith, R. I. 2016 A reduced-order deterministic model describing an intermittency route to combustion instability. Combust. Theor. Model. 20 (3), 441-456.

Shanbhogue, S. J. 2008. Dynamics of perturbed exothermic bluff-body flow-fields. Doctoral dissertation, Georgia Institute of Technology.

Singh, J., Belur Vishwanath, R., Chaudhuri, S. \& Sujith, R. I. 2017 Network structure of turbulent premixed flames. Chaos 27 (4), 043107.

Smith, D. A. \& Zukoski, E. 1985 Combustion instability sustained by unsteady vortex combustion. In 21st Joint Propulsion Conference, p. 1248.

Strahle, W. C. 1971 On combustion generated noise. J. Fluid Mech. 49 (2), 399-414. 
Strogatz, S. H. 2001 Exploring complex networks. Nature 410 (6825), 268-276.

Tachibana, S., Kanai, K., Yoshida, S., Suzuki, K. \& Sato, T. 2015 Combined effect of spatial and temporal variations of equivalence ratio on combustion instability in a low-swirl combustor. Proc. Combust. Inst. 35 (3), 3299-3308.

TAira, K., NAir, A. G. \& BRUnton, S. L. 2016 Network structure of two-dimensional decaying isotropic turbulence. J. Fluid Mech. 795, R2.

Tang, J., Scellato, S., Musolesi, M., Mascolo, C. \& Latora, V. 2010 Small-world behavior in time-varying graphs. Phys. Rev. E 81 (5), 055101.

Tony, J., Gopalakrishnan, E. A., Sreelekha, E. \& Sujith, R. I. 2015 Detecting deterministic nature of pressure measurements from a turbulent combustor. Phys. Rev. E 92 (6), 062902.

Tsonis, A. A. \& Roebber, P. J. 2004 The architecture of the climate network. Physica 333, 497-504.

Unni, V. R., Krishnan, A., Manikandan, R., George, N. B., Sujith, R. I., Marwan, N. \& KURTHS, J. 2018 On the emergence of critical regions at the onset of thermoacoustic instability in a turbulent combustor. Chaos 28 (6), 063125.

UnNi, V. R. \& SUJith, R. I. 2015 Multifractal characteristics of combustor dynamics close to lean blowout. J. Fluid Mech. 784, 30-50.

UnNi, V. R. \& SuJith, R. I. 2017 Flame dynamics during intermittency in a turbulent combustor. Proc. Combust. Inst. 36 (3), 3791-3798.

Valencia, M., Martinerie, J., Dupont, S. \& Chavez, M. 2008 Dynamic small-world behavior in functional brain networks unveiled by an event-related networks approach. Phys. Rev. E 77 (5), 050905.

Watts, D. J. \& Strogatz, S. H. 1998 Collective dynamics of 'small-world' networks. Nature 393 (6684), 440-442.

Weber, J. K. \& PAnde, V. S. 2015 Percolation-like phase transitions in network models of protein dynamics. J. Chem. Phys. 142 (21), 215105.

Zhang, Y., ZhaO, D., ZhANG, J., XiOng, R. \& GAO, W. 2011 Interpolation-dependent image downsampling. IEEE Trans. Image Process. 20 (11), 3291-3296. 